\title{
General multifractal analysis of local entropies
}

\author{
by
}

Floris Takens and Evgeny Verbitski (Groningen)

\begin{abstract}
We address the problem of the multifractal analysis of local entropies for arbitrary invariant measures. We obtain an upper estimate on the multifractal spectrum of local entropies, which is similar to the estimate for local dimensions. We show that in the case of Gibbs measures the above estimate becomes an exact equality. In this case the multifractal spectrum of local entropies is a smooth concave function.

We discuss possible singularities in the multifractal spectrum and their relation to phase transitions.
\end{abstract}

1. Introduction. The main problem of multifractal analysis is the description of local singularities of measures. Historically, multifractal analysis was mainly concerned with the study of local (pointwise) dimensions of a Borel measure $\mu$ (provided the limit exists):

$$
d_{\mu}(x)=\lim _{\varepsilon \rightarrow 0} \frac{\log \mu(\mathcal{B}(x, \varepsilon))}{\log \varepsilon},
$$

where $\mathcal{B}(x, \varepsilon)$ is an open $\varepsilon$-neighborhood of $x$. The purpose is to describe the sets of points with a given pointwise dimension. For this the notion of the multifractal spectrum (sometimes called the spectrum of singularities) $f(\alpha)$ was introduced in [10]. This spectrum is the function

$$
f(\alpha)=\operatorname{dim}_{\mathrm{H}}\left(\left\{x: d_{\mu}(x)=\alpha\right\}\right),
$$

where $\operatorname{dim}_{\mathrm{H}}$ is the Hausdorff dimension.

One can generalize this approach even further $[2,17]$, as we shall explain now. Suppose we are interested in some local (i.e., pointwise) characteristic of a measure or a dynamical system. We can think of it as a function $F: X \rightarrow \mathbb{R}$ $\left(F\right.$ comes in place of $d_{\mu}$ ). There is a natural multifractal decomposition of the state space $X$ into the level sets of $F$ :

$$
X=\bigcup_{\alpha \in \mathbb{R}} K_{\alpha}^{F},
$$

2000 Mathematics Subject Classification: 28A80, 37A35, 37D25. 
where $K_{\alpha}^{F}=\{x \in X: F(x)=\alpha\}$. We may even allow the situation where $F$ is not defined everywhere in $X$. Then we have to add to the multifractal decomposition (1.2) the set

$$
K^{F}=\{x \in X: F(x) \text { is not defined }\} .
$$

A multifractal spectrum $\mathcal{E}$ is by definition a function which assigns "sizes" to the level sets:

$$
\mathcal{E}(\alpha)=\mathcal{F}\left(K_{\alpha}^{F}\right), \quad \alpha \in \mathbb{R},
$$

where $\mathcal{F}$ is some set function, i.e., $\mathcal{F}$ is defined on subsets of $X$, and has the property that $\mathcal{F}\left(Z_{1}\right) \leq \mathcal{F}\left(Z_{2}\right)$ for $Z_{1} \subseteq Z_{2}$. The set function $\mathcal{F}$ plays the role of the Hausdorff dimension in (1.1).

So such a multifractal spectrum is a real function on $\mathbb{R}$ depending on a pair $(F, \mathcal{F})$ as introduced above. Therefore, the multifractal spectrum defined by (1.1) is the multifractal spectrum for local (pointwise) dimensions and the Hausdorff dimension, abbreviated to the multifractal spectrum for local dimensions.

There are a few local characteristics, relevant from the dynamical point of view: local dimension (discussed above), local entropies, Lyapunov exponents. There is also a choice of set functions: Hausdorff dimension, packing dimension [7], topological entropy for non-compact sets $[4,17]$. All these multifractal spectra provide a description of various aspects of dynamical systems (chaoticity, sensitive dependence, etc.). These spectra are invariant under smooth conjugations (even under homeomorphisms with bounded distortion). This is very important in relation to the notion of multifractal rigidity, introduced in [2].

In the present paper we study the multifractal spectrum for local entropies. Consider a topological dynamical system $(X, f, \mu)$, where $X$ is a compact metric space and $f: X \rightarrow X$ is a continuous map preserving the Borel probability measure $\mu$. Define the local entropy at a point $x$ as follows:

$$
h_{\mu}(f, x)=\lim _{\varepsilon \rightarrow 0} \lim _{n \rightarrow 0}-\frac{1}{n} \log \mu\left(\mathcal{B}_{n}(x, \varepsilon)\right),
$$

where $\mathcal{B}_{n}(x, \varepsilon)=\left\{y \in X: d\left(f^{i}(x), f^{i}(y)\right)<\varepsilon\right.$ for $\left.i=0, \ldots, n-1\right\}, \varepsilon>0$. We define the multifractal spectrum for local entropies and the topological entropy of non-compact sets as

$$
\mathcal{E}(\alpha)=h_{\text {top }}\left(f,\left\{x: h_{\mu}(f, x)=\alpha\right\}\right),
$$

where $h_{\text {top }}(f, Z)$ is the topological entropy of $Z$. We abbreviate this to the multifractal spectrum for local entropies. The precise definition is given below, but for the time being one could think of $h_{\text {top }}(f, Z)$ as a dynamical analogue of the Hausdorff dimension.

The two multifractal spectra, namely, for local dimensions and for local entropies, contain complementary information about the invariant measure $\mu$ 
and the dynamical system $f$, as one can see in the example of a skew tent map: for $p \in(0,1)$, put

$$
f_{p}(x)= \begin{cases}x / p & \text { for } x \in[0, p), \\ (1-x) /(1-p) & \text { for } x \in[p, 1] .\end{cases}
$$

For the Lebesgue measure, which is invariant, the dimension spectrum is trivial, since $d_{\mu}(x)=1$ for all $x \in[0,1]$. However, for $p \neq 1 / 2$, the Lebesgue measure has a non-trivial spectrum for local entropies. This will follow from later considerations.

Apart from pointwise dimensions and entropies also global dimensions and entropies are defined. There are a few known characteristics of such type: Rényi dimensions, Hentschel-Procaccia dimension, correlation entropies. For $q \neq 1$ they are given by the following formulas:

1) Generalized Rényi dimensions:

$$
D_{q}=\frac{1}{q-1} \lim _{\varepsilon \rightarrow 0} \frac{1}{\log \varepsilon} \log \sum_{i} p_{i}^{q},
$$

where $p_{i}=\mu(i$ th cell of a partition by cubes of size $\varepsilon)$;

2) Hentschel-Procaccia dimensions:

$$
\widetilde{D}_{q}=\frac{1}{q-1} \lim _{\varepsilon \rightarrow 0} \frac{1}{\log \varepsilon} \log \int \mu(\mathcal{B}(x, \varepsilon))^{q-1} d \mu(x) ;
$$

3) Correlation entropies:

$$
H_{\mu}(f, q)=\lim _{\varepsilon \rightarrow 0} \varliminf_{n \rightarrow \infty}-\frac{1}{(q-1) n} \log \int \mu\left(\mathcal{B}_{n}(x, \varepsilon)\right)^{q-1} d \mu .
$$

The corresponding definitions for $q=1$ are obtained by the continuity of the pre-limit quantities. Under quite mild assumptions, the Rényi and HentschelProcaccia dimensions coincide $[9,17]$.

So we consider the generalized dimensions (entropies) of a measure space $(X, \mu)$ (resp. dynamical system $(X, f, \mu))$ as functions which assign to every $q \in \mathbb{R}$ the dimension (entropy) $D_{q}$ (resp. $H_{q}$ ).

The standard ("folklore" according to [16]) result of multifractal analysis establishes that, under some conditions, a multifractal spectrum for dimensions is a smooth concave function on a certain interval and is equal to the Legendre transform of the generalized dimensions. In this case we say that the multifractal formalism is valid. However, it is not always the case. There are numerous examples when the multifractal formalism fails.

It has been shown in [16] that even in the case when the multifractal formalism for local dimensions is not valid, one obtains an upper estimate on the multifractal spectrum by taking the Legendre transform of the generalized dimensions. 
In the present paper we obtain similar results for the multifractal analysis of local (pointwise) entropies. In our case, the spectrum of correlation entropies plays the role of Hentschel-Procaccia and Rényi spectra of dimensions.

Finally, we present examples of chaotic dynamical systems for which the methods developed give a complete or partial description of the multifractal spectrum of local entropies.

2. Local entropy, Brin-Katok formula. Consider a compact metric space $(X, d)$. Let $f: X \rightarrow X$ be a continuous map and $\mu$ an invariant nonatomic Borel measure. Without loss of generality we may assume that $\mu$ is positive on open sets. In this case we define the lower (resp. upper) local (pointwise) entropies as follows:

$$
\begin{aligned}
& \underline{h}_{\mu}(f, x)=\lim _{\varepsilon \rightarrow 0} \frac{\lim _{n \rightarrow \infty}}{n \rightarrow}-\frac{1}{n} \log \mu\left(\mathcal{B}_{n}(x, \varepsilon)\right), \\
& \bar{h}_{\mu}(f, x)=\lim _{\varepsilon \rightarrow 0} \varlimsup_{n \rightarrow \infty}-\frac{1}{n} \log \mu\left(\mathcal{B}_{n}(x, \varepsilon)\right),
\end{aligned}
$$

where $\mathcal{B}_{n}(x, \varepsilon)=\left\{y \in X: d\left(f^{i}(x), f^{i}(y)\right)<\varepsilon\right.$ for $\left.i=0, \ldots, n-1\right\}$. Note that the limits in $\varepsilon$ exist by monotonicity.

We say that the local entropy exists at $x$ if

$$
\underline{h}_{\mu}(f, x)=\bar{h}_{\mu}(f, x) .
$$

In this case the common value will be denoted by $h_{\mu}(f, x)$.

The following well-known result establishes the existence of local entropies.

Theorem 2.1 (Brin-Katok formula, [6]). Let $f: X \rightarrow X$ be a continuous map on a compact metric space $(X, d)$ preserving a non-atomic Borel measure $\mu$. Then

(1) for $\mu$-a.e. $x \in X$ the local entropy exists, i.e.,

$$
h_{\mu}(f, x)=\underline{h}_{\mu}(f, x)=\bar{h}_{\mu}(f, x)
$$

(2) $h_{\mu}(f, x)$ is an $f$-invariant function of $x$, and

$$
\int h_{\mu}(f, x) d \mu=h_{\mu}(f)
$$

where $h_{\mu}(f)$ is the measure-theoretic entropy of $f$.

REMARK. If $\mu$ is ergodic then $h_{\mu}(f, x)=h_{\mu}(f)$ for $\mu$-a.e. $x \in X$.

In this paper we study the multifractal spectrum of local entropies. For every $\alpha \geq 0$ consider the level set of local entropies:

$$
K_{\alpha}=\left\{x \in X: h_{\mu}(f, x)=\alpha\right\} .
$$


We define the multifractal spectrum of local entropies by

$$
\mathcal{E}(\alpha)=h_{\text {top }}\left(f, K_{\alpha}\right),
$$

where $h_{\text {top }}\left(f, K_{\alpha}\right)$ is the topological entropy of $f$ restricted to $K_{\alpha}$ (or simply, the topological entropy of $K_{\alpha}$ ). The precise definition and basic facts are given in the next section.

3. Topological entropy of non-compact sets. The notion of topological entropy of non-compact or non-invariant sets has been introduced by R. Bowen in [4]. In this section we recall an equivalent definition due to Ya. B. Pesin and B. S. Pitskel' [18] (see also [17]).

Suppose $f: X \rightarrow X$ is a continuous map of a compact metric space $(X, d)$. Let $\mathcal{U}=\left\{U_{1}, \ldots, U_{M}\right\}$ be a finite open cover of $X$. By definition, a string $\mathbf{U}$ is a sequence $U_{i_{1}} \ldots U_{i_{n}}$ with $i_{k} \in\{1, \ldots, M\}$; its length $n$ is denoted by $n(\mathbf{U})$. The collection of all strings of length $n$ is denoted by $W_{n}(\mathcal{U})$, and $W_{\geq n}(\mathcal{U})=\bigcup_{k \geq n} W_{k}(\mathcal{U})$. For each $\mathbf{U} \in W_{n}(\mathcal{U})$ define

$$
\begin{aligned}
X(\mathbf{U}) & =U_{i_{1}} \cap f^{-1} U_{i_{2}} \cap \ldots \cap f^{-n+1} U_{i_{n}} \\
& =\left\{x \in X: f^{k-1} x \in U_{i_{k}}, k=1, \ldots, n\right\} .
\end{aligned}
$$

We say that a collection $\Gamma$ of strings covers a set $Z \subseteq X$ if $Z \subseteq$ $\bigcup_{\mathbf{U} \in \Gamma} X(\mathbf{U})$.

For any real number $s$ and every collection $\Gamma$ of strings we introduce the free energy as follows:

$$
F(\Gamma, s)=\sum_{\mathbf{U} \in \Gamma} \exp (-n(\mathbf{U}) s)
$$

For a given $Z$ consider the infimum of free energies over all collections $\Gamma \subseteq W_{\geq n}$ which cover $Z$ :

$$
M(Z, \mathcal{U}, s, n)=\inf _{\Gamma \operatorname{covers} Z} F(\Gamma, s)
$$

and put

$$
M(Z, \mathcal{U}, s)=\lim _{n \rightarrow \infty} M(Z, \mathcal{U}, s, n) .
$$

There exists a unique value $\widehat{s}$ such that $M(Z, \cdot, \mathcal{U})$ jumps from $+\infty$ to 0 :

$$
\begin{aligned}
h(Z, \mathcal{U}):=\widehat{s} & =\sup \{s: M(Z, \mathcal{U}, s)=+\infty\} \\
& =\inf \{s: M(Z, \mathcal{U}, s)=0\} .
\end{aligned}
$$

Finally, one can show [17] that the following limit exists:

$$
h_{\text {top }}(f, Z):=\lim _{\operatorname{diam}(\mathcal{U}) \rightarrow 0} h(Z, \mathcal{U}) .
$$

Definition 3.1. The number $h_{\text {top }}(f, Z)$ is called the topological entropy of $f$ restricted to the set $Z$, or, simply, the topological entropy of $Z$. 
This definition of topological entropy is similar to the definition of Hausdorff dimension (the diameters of the covering open sets are replaced by $\exp (-n(\mathbf{U}))$, which could be understood as a "dynamical diameter" of $X(\mathbf{U}))$. Indeed, these definitions are particular cases of the so-called Carathéodory construction [17] and, therefore, have similar properties.

THEOREM 3.2 (see [17]). The topological entropy as defined above has the following properties:

(1) $h_{\text {top }}\left(f, Z_{1}\right) \leq h_{\text {top }}\left(f, Z_{2}\right)$ for any $Z_{1} \subseteq Z_{2} \subseteq X$;

(2) $h_{\mathrm{top}}(f, Z)=\sup _{i} h_{\mathrm{top}}\left(f, Z_{i}\right)$ where $Z=\bigcup_{i=1}^{\infty} Z_{i}, Z_{i} \subseteq X$; $h_{\mu}(f)$

(3) if $\mu$ is an invariant measure such that $\mu(Z)=1$ then $h_{\mathrm{top}}(f, Z) \geq$

4. $(q, \mu)$-entropy of non-compact or non-invariant sets. Entropy doubling condition. In this section following the ideas of [16] and a formalism from [17] we introduce the entropy-related dimension characteristic $h_{\mu}(f, q, Z)$, which we call the $(q, \mu)$-entropy of $f$ restricted to $Z$, or simply, the $(q, \mu)$-entropy of $Z$, when there is no confusion about the dynamics $f$.

This definition requires a few steps and goes along the lines of the definition of topological entropy from the previous section.

For any at most countable collection $\mathcal{G}=\left\{\mathcal{B}_{n_{i}}\left(x_{i}, \varepsilon\right)\right\}_{i}$ and any $q, t \in \mathbb{R}$ define the $(q, t)$-free energy of $\mathcal{G}$ by

$$
F_{\mu}(\mathcal{G}, q, t)=\sum_{i} \mu\left(\mathcal{B}_{n_{i}}\left(x_{i}, \varepsilon\right)\right)^{q} \exp \left(-t n_{i}\right)
$$

For any given set $Z \subseteq X, Z \neq \emptyset$, and numbers $q, t \in \mathbb{R}, \varepsilon>0, N \in \mathbb{N}$ put

$$
M_{\mu}^{c}(Z, q, t, \varepsilon, N)=\inf _{\mathcal{G}} F_{\mu}(\mathcal{G}, q, t)
$$

where the infimum is taken over all finite or countable collections $\mathcal{G}=$ $\left\{\mathcal{B}_{n_{i}}\left(x_{i}, \varepsilon\right)\right\}_{i}$ with $x_{i} \in Z$ and $n_{i} \geq N$ such that $Z \subseteq \bigcup_{i} \mathcal{B}_{n_{i}}\left(x_{i}, \varepsilon\right)$. To complete the definition we put

$$
M_{\mu}^{c}(\emptyset, q, t, \varepsilon, N)=0
$$

for any $q, t, \varepsilon$ and $N$.

The quantities $M_{\mu}^{c}(Z, q, t, \varepsilon, N)$ are non-decreasing in $N$, hence the following limit exists:

$$
M_{\mu}^{c}(Z, q, t, \varepsilon)=\lim _{N \rightarrow \infty} M_{\mu}^{c}(Z, q, t, \varepsilon, N)=\sup _{N>1} M_{\mu}^{c}(Z, q, t, \varepsilon, N) .
$$

Since we consider covers with centers in a given set, the quantities $M_{\mu}^{c}(Z, q, t, \varepsilon)$ are not necessarily monotonic with respect to the set $Z$. We enforce monotonicity by putting

$$
M_{\mu}(Z, q, t, \varepsilon)=\sup _{Z^{\prime} \subseteq Z} M_{\mu}^{c}\left(Z^{\prime}, q, t, \varepsilon\right) .
$$


Lemma 4.1. For any $t \in \mathbb{R}$ the set function $M_{\mu}(\cdot, q, t, \varepsilon)$ has the following properties:

(i) $M_{\mu}(\emptyset, q, t, \varepsilon)=0$;

(ii) $M_{\mu}\left(Z_{1}, q, t, \varepsilon\right) \leq M_{\mu}\left(Z_{2}, q, t, \varepsilon\right)$ for any $Z_{1} \subseteq Z_{2}$;

(iii) $M_{\mu}\left(\bigcup_{i} Z_{i}, q, t, \varepsilon\right) \leq \sum_{i} M_{\mu}\left(Z_{i}, q, t, \varepsilon\right)$ for any $Z_{i} \subseteq X$.

REMARK. In other words, $M(\cdot, q, t, \varepsilon)$ is an outer measure [17]. The role of $M(Z, q, t, \varepsilon)$ is similar to that of the $s$-dimensional Hausdorff measure in the definition of Hausdorff dimension.

Lemma 4.2. There exists a critical value $h_{\mu}(f, q, Z, \varepsilon) \in[-\infty,+\infty]$ such that

$$
M_{\mu}(Z, q, t, \varepsilon)= \begin{cases}\infty, & t<h_{\mu}(f, q, Z, \varepsilon) \\ 0, & t>h_{\mu}(f, q, Z, \varepsilon)\end{cases}
$$

Proof. The statement is a simple consequence of the following standard observation. If $M_{\mu}(Z, q, \widehat{t}, \varepsilon)<\infty$ for some $\widehat{t}$, then $M_{\mu}(Z, q, t, \varepsilon)=0$ for all $t>\widehat{t}$. If $M_{\mu}(Z, q, \widehat{t}, \varepsilon)>0$ for some $\widehat{t}$, then $M_{\mu}(Z, q, t, \varepsilon)=\infty$ for all $t<\widehat{t}$.

LEMMA 4.3. The following holds:

(i) $h_{\mu}(f, q, \emptyset, \varepsilon)=-\infty$;

(ii) $h_{\mu}\left(f, q, Z_{1}, \varepsilon\right) \leq h_{\mu}\left(f, q, Z_{2}, \varepsilon\right)$ for $Z_{1} \subseteq Z_{2}$;

(iii) $h_{\mu}\left(f, q, \bigcup_{i} Z_{i}, \varepsilon\right)=\sup _{i} h_{\mu}\left(f, q, Z_{i}, \varepsilon\right)$, where $Z_{i} \subseteq X, i=1,2, \ldots$

Proof. The first statement follows from the fact that $M_{\mu}(\emptyset, q, t, \varepsilon)=0$ for any $q, t$. The second statement is a direct consequence of the corresponding statement from the previous lemma.

Note that from (ii) one concludes that $h_{\mu}\left(f, q, \bigcup_{i} Z_{i}, \varepsilon\right) \geq h_{\mu}\left(f, q, Z_{i}, \varepsilon\right)$ for every $i$. Hence $h_{\mu}\left(f, q, \bigcup_{i} Z_{i}, \varepsilon\right) \geq \sup _{i} h_{\mu}\left(f, q, Z_{i}, \varepsilon\right)$. On the other hand, suppose that $h_{\mu}\left(f, q, Z_{i}, \varepsilon\right)<t$ for all $i$. Thus $M_{\mu}\left(Z_{i}, q, t, \varepsilon\right)=0$ for every $i$ and therefore from the previous lemma one has $M_{\mu}\left(\bigcup_{i} Z_{i}, q, t, \varepsilon\right)=0$. This means that $h_{\mu}\left(f, q, \bigcup_{i} Z_{i}, \varepsilon\right) \leq t$. Hence, one obtains $h_{\mu}\left(f, q, \bigcup_{i} Z_{i}, \varepsilon\right)$ $\leq \sup _{i} h_{\mu}\left(f, q, Z_{i}, \varepsilon\right)$, and the result follows.

We are interested in the asymptotic behavior of local entropies as $\varepsilon \rightarrow 0$.

Definition 4.4. The $(q, \mu)$-entropy of $Z$ is

$$
h_{\mu}(f, q, Z)=\varlimsup_{\varepsilon \rightarrow 0} h_{\mu}(f, q, Z, \varepsilon) .
$$

Let us discuss the existence of the limit with respect to $\varepsilon$ in the definition above.

If $q \leq 0$, then for $\varepsilon_{1}>\varepsilon_{2}>0$ one has $h_{\mu}\left(f, q, Z, \varepsilon_{1}\right) \geq h_{\mu}\left(f, q, Z, \varepsilon_{2}\right)$ and the limit in (4.2) exists.

Indeed, let $\mathcal{G}=\left\{\mathcal{B}_{n_{i}}\left(x_{i}, \varepsilon_{2}\right)\right\}$ be a centered cover of $Z$. Then obviously $\mathcal{G}^{\prime}=\left\{\mathcal{B}_{n_{i}}\left(x_{i}, \varepsilon_{1}\right)\right\}$ is a cover of $Z$ as well and $F_{\mu}(\mathcal{G}, q, t) \geq F_{\mu}\left(\mathcal{G}^{\prime}, q, t\right)$. Therefore $M_{\mu}\left(Z, q, t, \varepsilon_{2}\right) \geq M_{\mu}\left(Z, q, t, \varepsilon_{1}\right)$, and hence the limit in (4.2) exists. 
In general, for $q>0$ there is no monotonicity with respect to $\varepsilon$. However, under an additional assumption on the invariant measure $\mu$ one obtains the monotonic behavior with respect to $\varepsilon$. We formulate this property in the following definition.

DEFINITION 4.5. We say that an invariant measure $\mu$ satisfies the entropy doubling condition if for every sufficiently small $\varepsilon>0$,

$$
C(\varepsilon):=\sup _{n} \sup _{x} \frac{\mu\left(\mathcal{B}_{n}(x, \varepsilon)\right)}{\mu\left(\mathcal{B}_{n}(x, \varepsilon / 2)\right)}<\infty .
$$

REMARK. The constant $1 / 2$ in the previous definition is not crucial. It is easy to see that if a measure $\mu$ satisfies the entropy doubling condition, then

$$
\sup _{n} \sup _{x} \frac{\mu\left(\mathcal{B}_{n}(x, \varepsilon)\right)}{\mu\left(\mathcal{B}_{n}(x, \varepsilon / a)\right)}<\infty
$$

for any $a>1$ and all sufficiently small $\varepsilon>0$.

Now we show the existence of the limit in (4.2) for measures satisfying the entropy doubling condition.

Indeed, assume that $\mu$ satisfies the entropy doubling condition for all $\varepsilon \in\left(0, \varepsilon_{0}\right)$. Take some $\varepsilon_{1}, \varepsilon_{2} \in\left(0, \varepsilon_{0}\right)$ and let $a=\varepsilon_{1} / \varepsilon_{2}>1$. From the previous remark we conclude that there exists $\widetilde{C}=\widetilde{C}(a)<\infty$ such that

$$
\frac{\mu\left(\mathcal{B}_{n}\left(x, \varepsilon_{1}\right)\right)}{\mu\left(\mathcal{B}_{n}\left(x, \varepsilon_{1} / a\right)\right)}=\frac{\mu\left(\mathcal{B}_{n}\left(x, \varepsilon_{1}\right)\right)}{\mu\left(\mathcal{B}_{n}\left(x, \varepsilon_{2}\right)\right)}<\widetilde{C}(a)
$$

for all $n$. Now let $\mathcal{G}=\left\{\mathcal{B}_{n_{i}}\left(x_{i}, \varepsilon_{2}\right)\right\}$ be a centered cover of $Z$. Then $\mathcal{G}^{\prime}=$ $\left\{\mathcal{B}_{n_{i}}\left(x_{i}, \varepsilon_{1}\right)\right\}$ is a centered cover of $Z$ as well and $F_{\mu}(\mathcal{G}, q, t) \geq \widetilde{C}^{-q} F_{\mu}\left(\mathcal{G}^{\prime}, q, t\right)$. Thus $M_{\mu}\left(Z, q, t, \varepsilon_{2}\right) \geq \widetilde{C}^{-q} M_{\mu}\left(Z, q, t, \varepsilon_{1}\right)$, and therefore $h_{\mu}\left(f, q, Z, \varepsilon_{2}\right) \geq$ $h_{\mu}\left(f, q, Z, \varepsilon_{1}\right)$. Hence, the limit in (4.2) exists.

Finally, note that all the statements of Lemma 4.3 remain true for $h_{\mu}(f, Z, q)$ as well.

5. Coincidence of the topological entropy and $(0, \mu)$-entropy. We begin by making a simple observation which we will often exploit later.

Let $\mathcal{U}$ be a finite open cover of a compact metric space $(X, d)$. Then there exists a positive number $\delta(\mathcal{U})$, which is called a Lebesgue number of $\mathcal{U}$, such that for any open set $V \subseteq X$ with $\operatorname{diam}(V)<\delta(\mathcal{U})$ there exists an element $U$ of $\mathcal{U}$ containing $V$.

Suppose now that $\varepsilon_{1}<\delta(\mathcal{U}) / 2$. Then for any $x \in X$ and each $n \in \mathbb{N}$, there exists a string $\mathbf{U}_{x}=U_{i_{1}} \ldots U_{i_{n}}$ of length $n$ such that

$$
\mathcal{B}_{n}\left(x, \varepsilon_{1}\right) \subseteq X\left(\mathbf{U}_{x}\right) .
$$

We can obtain this string by choosing some $U_{i_{k}}$ from $\mathcal{U}$, which contains $\mathcal{B}\left(f^{k-1} x, \varepsilon_{1}\right)$. This is possible because $\varepsilon_{1}<\delta(\mathcal{U}) / 2$. 
For an arbitrary string $\mathbf{U}=U_{i_{1}} \ldots U_{i_{n}}$ take some $x \in X(\mathbf{U})=U_{i_{1}} \cap$ $f^{-1} U_{i_{2}} \cap \ldots \cap f^{-n+1} U_{i_{n}}$. Then

$$
X(\mathbf{U}) \subseteq \mathcal{B}_{n(\mathbf{U})}\left(x, \varepsilon_{2}\right)
$$

for any $\varepsilon_{2}>2 \operatorname{diam}(\mathcal{U})$.

The measure $\mu$ does not actually participate in the definition of $h_{\mu}(f, q, Z)$ in the case of $q=0$. This leads to the following fact.

Lemma 5.1. For any set $Z \subseteq X$ one has $h_{\mu}(f, 0, Z)=h_{\text {top }}(f, Z)$.

Proof. If $Z=\emptyset$ the statement is obvious, since both sides are equal to $-\infty$. Suppose now that $Z \neq \emptyset$. We start by showing $h_{\text {top }}(f, Z) \leq h_{\mu}(f, 0, Z)$. Let $\mathcal{U}$ be an arbitrary finite open cover of $X$ and $\varepsilon<\delta(\mathcal{U}) / 2$. Consider an arbitrary collection $\left.\mathcal{G}=\left\{\mathcal{B}_{\backslash}\left(\S_{\rangle}, \varepsilon\right)\right\}\right\rangle$ such that $x_{i} \in Z$ and $Z \subseteq \bigcup_{i} \mathcal{B}_{n_{i}}\left(x_{i}, \varepsilon\right)$.

For any $i$, using (5.1), we choose some string $\mathbf{U}(i)$ of length $n(\mathbf{U}(i))=n_{i}$ such that $\mathcal{B}_{n_{i}}\left(x_{i}, \varepsilon\right) \subseteq X(\mathbf{U}(i))$. Let $\Gamma_{\mathcal{G}}=\{\mathbf{U}(i)\}$. Obviously, $\Gamma_{\mathcal{G}}$ covers $Z$ and

$$
F_{\mu}(\mathcal{G}, 0, t)=\sum_{i} \exp \left(-t n_{i}\right)=\sum_{\mathbf{U} \in \Gamma_{\mathcal{G}}} \exp (-t n(\mathbf{U}))=F(\Gamma, t) .
$$

Since $\mathcal{G}$ is an arbitrary covering we conclude that

$$
M_{\mu}^{c}(Z, 0, t, \varepsilon, n)=\inf _{\mathcal{G} \operatorname{covers} Z} F_{\mu}(\mathcal{G}, 0, t) \geq \inf _{\Gamma \operatorname{covers} Z} F(\Gamma, t)=M(Z, \mathcal{U}, t, n) .
$$

Taking limits as $n \rightarrow \infty$ we conclude that

$$
M(Z, \mathcal{U}, t) \leq M_{\mu}^{c}(Z, 0, t, \varepsilon) \leq M_{\mu}(Z, 0, t, \varepsilon) .
$$

Therefore, $h(Z, \mathcal{U}) \leq h_{\mu}(f, 0, Z, \varepsilon)$ for any $\varepsilon<\delta(\mathcal{U}) / 2$. Since

$$
h_{\text {top }}(f, Z)=\lim _{\operatorname{diam}(\mathcal{U}) \rightarrow 0} h(Z, \mathcal{U})
$$

we conclude that $h_{\text {top }}(f, Z) \leq \overline{\lim }_{\varepsilon \rightarrow 0} h_{\mu}(f, 0, Z, \varepsilon)=h_{\mu}(f, 0, Z)$.

Let us now show the opposite inequality. Assume $h_{\mu}(f, 0, Z)-h_{\text {top }}(f, Z)$ $>3 \gamma>0$. Then there exists $\varepsilon>0$ such that

$$
h_{\mu}(f, 0, Z, \varepsilon)-h_{\mathrm{top}}(f, Z)>2 \gamma .
$$

Since $h_{\text {top }}(f, Z)=\lim _{\operatorname{diam}(\mathcal{U}) \rightarrow 0} h(Z, \mathcal{U})$ there exists a finite open cover $\mathcal{U}$ with sufficiently small diameter (in particular, $\operatorname{diam}(\mathcal{U})<\varepsilon / 2$ ) such that

$$
h_{\mu}(f, 0, Z, \varepsilon)-h(Z, \mathcal{U})>\gamma>0 .
$$

Let $Z^{\prime}$ be an arbitrary subset of $Z$ and $\Gamma$ an arbitrary collection of strings covering $Z^{\prime}$. We may assume that $X(\mathbf{U}) \cap Z^{\prime} \neq \emptyset$ for any $\mathbf{U} \in \Gamma$. Otherwise we just delete those strings and obtain a smaller collection of strings, which still covers $Z^{\prime}$.

For any $\mathbf{U} \in \Gamma$ choose an arbitrary $x(\mathbf{U}) \in X(\mathbf{U}) \cap Z^{\prime}$. Then by (5.2) we get

$$
X(\mathbf{U}) \subseteq \mathcal{B}_{n(\mathbf{U})}(x(\mathbf{U}), \varepsilon) .
$$


Therefore, the collection $\mathcal{G}=\left\{\mathcal{B}_{n(\mathbf{U})}(x(\mathbf{U}), \varepsilon)\right\}$ is a centered cover of $Z^{\prime}$. Since their free energies $F(\Gamma, s)$ and $F_{\mu}(\mathcal{G}, 0, s)$ are the same we obtain

$$
M_{\mu}^{c}\left(Z^{\prime}, 0, s, \varepsilon\right) \leq M\left(Z^{\prime}, \mathcal{U}, s\right) \leq M(Z, \mathcal{U}, s) .
$$

The last inequality holds due to the monotonicity of $M(\cdot, \mathcal{U}, s)$ with respect to the first argument. As a result we obtain

$$
M_{\mu}(Z, 0, s, \varepsilon)=\sup _{Z^{\prime} \subseteq Z} M_{\mu}^{c}\left(Z^{\prime}, 0, s, \varepsilon\right) \leq M(Z, \mathcal{U}, s) .
$$

This implies $h_{\mu}(f, 0, Z, \varepsilon) \leq h(Z, \mathcal{U})$, contradicting (5.3) and hence the assumption $h_{\mu}(f, 0, Z)>h_{\text {top }}(f, Z)$ as well.

6. Relation between the topological and $(q, \mu)$-entropies of the level sets $K_{\alpha}$. In this section we are going to establish that actually for any $\alpha \geq 0$ and every $q \in \mathbb{R}$ one has

$$
h_{\mathrm{top}}\left(f, K_{\alpha}\right)=q \alpha+h_{\mu}\left(f, q, K_{\alpha}\right) .
$$

On the intuitive level, one can explain the above equality as follows. Suppose $x \in K_{\alpha}$. Then for sufficiently large $n$ and sufficiently small $\varepsilon>0$ one has

$$
\mu\left(\mathcal{B}_{n}(x, \varepsilon)\right)^{q} \exp (-n t) \approx \exp (-n(q \alpha+t)) .
$$

Using this observation for any centered covering $\mathcal{G}=\left\{\mathcal{B}_{n_{i}}\left(x_{i}, \varepsilon\right)\right\}$ of $K_{\alpha}$ we can construct some collection of strings $\Gamma$ such that

$$
F_{\mu}(\mathcal{G}, q, t) \approx F(\Gamma, q \alpha+t) .
$$

The same is true in the opposite direction: for every collection of strings $\Gamma$, we can choose a centered covering $\mathcal{G}$ such that (6.2) holds. This implies that the outer measures $M\left(K_{\alpha}, q \alpha+t\right)$ and $M_{\mu}\left(K_{\alpha}, q, t\right)$, involved in the definition of $h_{\text {top }}\left(f, K_{\alpha}\right)$ and $h_{\mu}\left(f, q, K_{\alpha}\right)$, are simultaneously equal to $\infty$ or 0 . As a result we obtain the corresponding relation between $h_{\text {top }}\left(f, K_{\alpha}\right)$ and $h_{\mu}\left(f, q, K_{\alpha}\right)$. The proof is somewhat similar to the proof of the equality $h_{\text {top }}(f, Z)=h_{\mu}(f, 0, Z)$ for all $Z$ from the previous section. However, some additional work is required since for points with local entropy $\alpha$, the convergence of the corresponding limit to $\alpha$ can be non-uniform. Therefore instead of $K_{\alpha}$ we will consider a monotonic sequence of subsets of $K_{\alpha}$ which exhausts $K_{\alpha}$ and for which the convergence of $\left(-\log \mu\left(\mathcal{B}_{n}(x, \varepsilon)\right)\right) / n$ to $\alpha$ is uniform. Both the topological and $(q, \mu)$-entropies have the following property: for any set $Z$ and any sequence $Z_{i} \subseteq Z$ such that $Z_{i} \subseteq Z_{i+1}$ and $Z=\bigcup_{i} Z_{i}$ one has

$$
h_{\mathrm{top}}(f, Z)=\lim _{i \rightarrow \infty} h_{\mathrm{top}}\left(f, Z_{i}\right), \quad h_{\mu}(f, q, Z)=\lim _{i \rightarrow \infty} h_{\mu}\left(f, q, Z_{i}\right) .
$$

Thus, if we establish some relation (similar to (6.1)) between $h_{\text {top }}\left(f, Z_{i}\right)$ and $h_{\mu}\left(f, q, Z_{i}\right)$, we will obtain a similar relation for $h_{\text {top }}(f, Z)$ and $h_{\mu}(f, q, Z)$ as well. 
Now, we make the above arguments rigorous.

Consider $\alpha \geq 0$ and the corresponding level set

$$
\begin{aligned}
K_{\alpha}=\{x \in X: & \left.h_{\mu}(f, x)=\alpha\right\} \\
= & \left\{x \in X: \lim _{\varepsilon \rightarrow 0} \varliminf_{n \rightarrow \infty}-\frac{1}{n} \log \mu\left(\mathcal{B}_{n}(x, \varepsilon)\right)\right. \\
& \left.=\lim _{\varepsilon \rightarrow 0} \varlimsup_{n \rightarrow \infty}-\frac{1}{n} \log \mu\left(\mathcal{B}_{n}(x, \varepsilon)\right)=\alpha\right\} .
\end{aligned}
$$

Choose some monotonic sequence $\varepsilon_{M} \rightarrow 0$ as $M \rightarrow \infty$. This sequence will be fixed for the rest of this section. Let $\delta>0$ and put (we omit the $\delta$-dependence in the notation)

$$
K_{\alpha, M}=\left\{x \in K_{\alpha}: \alpha-\delta<\varliminf_{n \rightarrow \infty}-\frac{1}{n} \log \mu\left(\mathcal{B}_{n}\left(x, \varepsilon_{M}\right)\right)\right\} .
$$

Obviously, $K_{\alpha, M} \subseteq K_{\alpha, M+1}$ and $K_{\alpha}=\bigcup_{M} K_{\alpha, M}$.

Note that due to the monotonicity of $-\log \mu\left(\mathcal{B}_{n}(x, \varepsilon)\right)$ in $\varepsilon$, for each $x \in$ $K_{\alpha}$ and every $\varepsilon>0$ one has

$$
\varlimsup_{n \rightarrow \infty}-\frac{1}{n} \log \mu\left(\mathcal{B}_{n}(x, \varepsilon)\right) \leq \alpha .
$$

For fixed $x \in K_{\alpha, M}$ there exists $N_{0}=N_{0}\left(x, \delta, \varepsilon_{M}\right)$ such that

$$
\alpha-\delta<-\frac{1}{n} \log \mu\left(\mathcal{B}_{n}\left(x, \varepsilon_{M}\right)\right)<\alpha+\delta
$$

for all $n>N_{0}$. Put

$$
K_{\alpha, M, N}=\left\{x \in K_{\alpha, M}: N_{0}=N_{0}\left(x, \delta, \varepsilon_{M}\right)<N\right\} .
$$

Again, it is easy to see that $K_{\alpha, M, N} \subseteq K_{\alpha, M, N+1}$ and $K_{\alpha, M}=\bigcup_{N} K_{\alpha, M, N}$.

Let $\mathcal{U}$ be a finite open cover of $X$. Using the properties of topological entropy we conclude that

$$
h\left(K_{\alpha}, \mathcal{U}\right)=\lim _{M \rightarrow \infty} \lim _{N \rightarrow \infty} h\left(K_{\alpha, M, N}, \mathcal{U}\right) .
$$

The following technical lemma establishes the relation between the quantities involved in the definitions of $h\left(K_{\alpha, M, N}, \mathcal{U}\right)$ and $h_{\mu}\left(f, q, K_{\alpha, M, N}, q, \varepsilon\right)$.

Lemma 6.1. Suppose $\mathcal{U}$ is an arbitrary open cover of $X$. Consider $K_{\alpha, M, N}$ for some $M, N \in \mathbb{N}$ such that $\varepsilon_{M}<\delta(\mathcal{U}) / 2$, where $\delta(\mathcal{U})$ is a Lebesgue number of $\mathcal{U}$. Then for $s \geq q \alpha+|q| \delta+t$ one has

$$
M\left(K_{\alpha, M, N}, \mathcal{U}, s\right) \leq M_{\mu}^{c}\left(K_{\alpha, M, N}, q, t, \varepsilon_{M}\right) .
$$

Proof. Suppose $n>N$ and $\mathcal{G}=\left\{\mathcal{B}_{n_{i}}\left(x_{i}, \varepsilon_{M}\right)\right\}_{i}$ is an arbitrary cover of $K_{\alpha, M, N}$ with $x_{i} \in K_{\alpha, M, N}$ such that $n_{i} \geq n$ for all $i$. 
By (5.1), for every $x_{i}$ there exists some string $\mathbf{U}(i)$ with $n(\mathbf{U}(i))=n_{i}$ such that $\mathcal{B}_{n_{i}}\left(x_{i}, \varepsilon_{M}\right) \subseteq X(\mathbf{U}(i))$ and hence

$$
K_{\alpha, M, N} \subseteq \bigcup_{i} \mathcal{B}_{n_{i}}\left(x_{i}, \varepsilon_{M}\right) \subseteq \bigcup_{i} X(\mathbf{U}(i)) .
$$

Therefore the collection $\Gamma_{\mathcal{G}}=\{\mathbf{U}(i)\}$ of strings covers $K_{\alpha, M, N}$.

Since $x_{i} \in K_{\alpha, M, N}$ for all $i$ and $n_{i} \geq n>N$ we have

$$
\exp \left(-(\alpha+\delta) n_{i}\right) \leq \mu\left(\mathcal{B}_{n_{i}}\left(x_{i}, \varepsilon_{M}\right)\right) \leq \exp \left(-(\alpha-\delta) n_{i}\right) .
$$

If $q \geq 0$ then $\mu\left(\mathcal{B}_{n_{i}}\left(x_{i}, \varepsilon\right)\right)^{q} \geq \exp \left(-q(\alpha+\delta) n_{i}\right)$ and

$$
\begin{aligned}
\sum_{i} \mu\left(\mathcal{B}_{n_{i}}\left(x_{i}, \varepsilon\right)\right)^{q} \exp \left(-t n_{i}\right) & \geq \sum_{i} \exp \left(-n_{i}(q \alpha+\delta+t)\right) \\
& \geq \sum_{\mathbf{U} \in \Gamma_{\mathcal{G}}} \exp (-n(\mathbf{U}) s) \\
& \geq M\left(K_{\alpha, M, N}, \mathcal{U}, s, n\right)
\end{aligned}
$$

for $s \geq q \alpha+q \delta+t$. Since $\mathcal{G}$ is an arbitrary centered covering we get

$$
M\left(K_{\alpha, M, N}, \mathcal{U}, s, n\right) \leq M_{\mu}^{c}\left(K_{\alpha, M, N}, q, t, \varepsilon, n\right)
$$

provided $s \geq q \alpha+q \delta+t$.

Similarly, if $q \leq 0$ then $\mu\left(\mathcal{B}_{n_{i}}\left(x_{i}, \varepsilon\right)\right)^{q} \geq \exp \left(-(\alpha-\delta) q n_{i}\right)$ and

$$
\begin{aligned}
\sum_{i} \mu\left(\mathcal{B}_{n_{i}}\left(x_{i}, \varepsilon\right)\right)^{q} \exp \left(-t n_{i}\right) & \geq \sum_{i} \exp \left(-n_{i}(q \alpha-q \delta+t)\right) \\
& \geq \sum_{\mathbf{U} \in \Gamma_{\mathcal{G}}} \exp (-n(\mathbf{U}) s) \\
& \geq M\left(K_{\alpha, M, N}, \mathcal{U}, s, n\right)
\end{aligned}
$$

for $s \geq q \alpha-q \delta+t$. Again, since $\mathcal{G}$ is an arbitrary centered covering we conclude that

$$
M\left(K_{\alpha, M, N}, \mathcal{U}, s, n\right) \leq M_{\mu}^{c}\left(K_{\alpha, M, N}, q, t, \varepsilon, n\right) \leq M_{\mu}\left(K_{\alpha, M, N}, q, t, \varepsilon, n\right) .
$$

Taking limits as $n$ tends to infinity we obtain the desired result.

Lemma 6.2. For any $\alpha \geq 0$ and any $q \in \mathbb{R}$ one has

$$
h_{\text {top }}\left(T, K_{\alpha}\right) \leq q \alpha+h_{\mu}\left(T, q, K_{\alpha}\right) .
$$

Proof. We may assume that $K_{\alpha} \neq \emptyset$. Otherwise, the statement is obvious, since both sides are equal to $-\infty$.

Suppose that the opposite is true: there exists $q \in \mathbb{R}$ such that

$$
\gamma=\frac{1}{4}\left(h_{\mathrm{top}}\left(f, K_{\alpha}\right)-q \alpha-h_{\mu}\left(f, q, K_{\alpha}\right)\right)>0 .
$$

Since

$$
h_{\text {top }}\left(T, K_{\alpha}\right)=\lim _{\operatorname{diam}(\mathcal{U}) \rightarrow 0} h\left(K_{\alpha}, \mathcal{U}\right)
$$


one can find a finite open cover $\mathcal{U}$ such that

$$
h\left(K_{\alpha}, \mathcal{U}\right)>q \alpha+h_{\mu}\left(T, q, K_{\alpha}\right)+3 \gamma .
$$

Let $\delta$ be an arbitrary positive number if $q=0$, and $\delta=\gamma /(2|q|)>0$ if $|q|>0$. Consider $K_{\alpha, M, N}$ defined in (6.3). Choose sufficiently large $M, N$ such that the following three conditions are satisfied:

$$
\begin{gathered}
h\left(K_{\alpha, M, N}, \mathcal{U}\right)>q \alpha+h_{\mu}\left(f, q, K_{\alpha}\right)+2 \gamma \\
\varepsilon_{M}<\delta(\mathcal{U}) / 2 \text { and } h_{\mu}\left(f, q, K_{\alpha}\right)+\gamma / 2>h_{\mu}\left(f, q, K_{\alpha}, \varepsilon_{M}\right) .
\end{gathered}
$$

This is possible because $h\left(K_{\alpha}, \mathcal{U}\right)=\lim _{M \rightarrow \infty} \lim _{N \rightarrow \infty} h\left(K_{\alpha, M, N}, \mathcal{U}\right), \varepsilon_{M} \rightarrow 0$ as $M \rightarrow \infty$ and $h_{\mu}\left(f, q, K_{\alpha}\right)=\varlimsup_{\lim _{\varepsilon \rightarrow 0}} h_{\mu}\left(f, q, K_{\alpha}, \varepsilon\right)$.

By the definition of $h\left(K_{\alpha, M, N}, \mathcal{U}\right)$ the inequality (6.4) implies

$$
M\left(K_{\alpha, M, N}, \mathcal{U}, q \alpha+h_{\mu}\left(T, q, K_{\alpha}\right)+2 \gamma\right)=\infty
$$

Using the estimates from Lemma 6.1 applied to $s=q \alpha+h_{\mu}\left(f, q, K_{\alpha}\right)+\gamma$ and $t=h_{\mu}\left(f, q, K_{\alpha}\right)+\gamma-|q| \delta$ we conclude that

$$
M_{\mu}^{c}\left(K_{\alpha, M, N}, q, h_{\mu}\left(f, q, K_{\alpha}\right)+\gamma-|q| \delta, \varepsilon_{M}\right)=\infty .
$$

Here we arrive at a contradiction with the assumption above. Indeed,

$$
\begin{aligned}
h_{\mu}\left(f, q, K_{\alpha}\right)+\gamma-|q| \delta \geq h_{\mu}\left(f, q, K_{\alpha}\right)+\gamma / 2 & >h_{\mu}\left(f, q, K_{\alpha}, \varepsilon_{M}\right) \\
& \geq h_{\mu}\left(f, q, K_{\alpha, M, N}, \varepsilon_{M}\right),
\end{aligned}
$$

and therefore one must have

$$
M_{\mu}\left(K_{\alpha, M, N}, q, h_{\mu}\left(f, q, K_{\alpha}\right)+\gamma-|q| \delta, \varepsilon_{M}\right)=0 .
$$

Let us prove the opposite inequality.

Fix some integers $M, N$ and let $Z \subseteq K_{\alpha, M, N}, Z \neq \emptyset$. Consider an arbitrary finite open cover $\mathcal{U}$ with $\operatorname{diam}(\mathcal{U})<\varepsilon_{M} / 2$. Choose any $n>N$ and let $\Gamma$ be an arbitrary collection of strings covering $Z$ with

$$
n(\Gamma)=\inf _{\mathbf{U} \in \Gamma} n(\mathbf{U}) \geq n>N .
$$

Without loss of generality we may assume that $X(\mathbf{U}) \cap Z \neq \emptyset$ for any $\mathbf{U} \in \Gamma$. Let $x(\mathbf{U})$ be an arbitrary point from this intersection. Then by (5.2) one has

$$
X(\mathbf{U}) \subseteq \mathcal{B}_{n(\mathbf{U})}\left(x(\mathbf{U}), \varepsilon_{M}\right)
$$

Hence the collection $\left\{\mathcal{B}_{n(\mathbf{U})}\left(x(\mathbf{U}), \varepsilon_{M}\right)\right\}$ is a centered cover of $Z$.

Since $x(\mathbf{U}) \in Z \subseteq K_{\alpha, M, N}$ and $n(\mathbf{U})>N$, one has

$$
\exp (-n(\mathbf{U})(\alpha+\delta)) \leq \mu\left(\mathcal{B}_{n(\mathbf{U})}(x(\mathbf{U}), \varepsilon)\right) \leq \exp (-n(\mathbf{U})(\alpha-\delta)) .
$$


Therefore for $q \geq 0$ one has

$$
\begin{aligned}
M_{\mu}^{c}\left(Z, q, t, \varepsilon_{M}, n\right) & \leq \sum_{\mathbf{U} \in \Gamma} \mu\left(\mathcal{B}_{n(\mathbf{U})}(x(\mathbf{U}), \varepsilon)\right)^{q} \exp (-n(\mathbf{U}) t) \\
& \leq \sum_{\mathbf{U} \in \Gamma} \exp (-n(\mathbf{U})(q \alpha-q \delta+t)) \\
& \leq \sum_{\mathbf{U} \in \Gamma} \exp (-n(\mathbf{U}) s) \quad \text { for } s \leq q \alpha-q \delta+t .
\end{aligned}
$$

Since $\Gamma$ is an arbitrary covering of $Z$ by strings of length at least $n$, for $s \leq$ $q \alpha-q \delta+t$, we get

$$
M_{\mu}^{c}\left(Z, q, t, \varepsilon_{M}, n\right) \leq M(Z, \mathcal{U}, s, n)
$$

Therefore,

$$
M_{\mu}^{c}\left(Z, q, t, \varepsilon_{M}\right) \leq M(Z, \mathcal{U}, s) \leq M\left(K_{\alpha, M, N}, \mathcal{U}, s\right)
$$

and

$$
M_{\mu}\left(K_{\alpha, M, N}, q, t, \varepsilon_{M}\right) \leq M\left(K_{\alpha, M, N}, \mathcal{U}, s\right) .
$$

For $q \leq 0$ from (6.6) we have

$$
\mu\left(\mathcal{B}_{n(\mathbf{U})}\left(x(\mathbf{U}), \varepsilon_{M}\right)\right)^{q} \leq \exp (-n(\mathbf{U}) q(\alpha+\delta)) .
$$

Hence

$$
\begin{aligned}
M_{\mu}^{c}\left(Z, q, t, \varepsilon_{M}, n\right) & \leq \sum_{\mathbf{U} \in \Gamma} \mu\left(\mathcal{B}_{n(\mathbf{U})}(x(\mathbf{U}), \varepsilon)\right)^{q} \exp (-n(\mathbf{U}) t) \\
& \leq \sum_{\mathbf{U} \in \Gamma} \exp (-n(\mathbf{U})(q \alpha+q \delta+t)) \\
& \leq \sum_{\mathbf{U} \in \Gamma} \exp (-n(\mathbf{U}) s) \quad \text { for } s \leq q \alpha+q \delta+t .
\end{aligned}
$$

Similarly to the case $q \geq 0$ we conclude that

$$
M_{\mu}\left(K_{\alpha, M, N}, q, t, \varepsilon_{M}\right) \leq M\left(K_{\alpha, M, N}, \mathcal{U}, s\right) .
$$

Thus, we proved the following result.

LEMma 6.3. Consider some $\delta>0$ and the corresponding set $K_{\alpha, M, N}$ for some $M, N$. Let $\mathcal{U}$ be any finite open cover with $\operatorname{diam}(\mathcal{U})<\varepsilon_{M} / 2$. Then for $s \leq q \alpha-|q| \delta+t$ one has

$$
M_{\mu}\left(K_{\alpha, M, N}, q, t, \varepsilon_{M}\right) \leq M\left(K_{\alpha, M, N}, \mathcal{U}, s\right) .
$$

Using arguments similar to the proof of Lemma 6.2 , we prove the following result.

Lemma 6.4. For any $\alpha \geq 0$ and any $q \in \mathbb{R}$ one has

$$
h_{\text {top }}\left(f, K_{\alpha}\right) \geq q \alpha+h_{\mu}\left(f, q, K_{\alpha}\right) .
$$

Combining the results of Lemmas 6.2 and 6.4 , we easily obtain 
Corollary 6.5. For any $\alpha \geq 0$ and any $q \in \mathbb{R}$ one has

$$
h_{\text {top }}\left(f, K_{\alpha}\right)=q \alpha+h_{\mu}\left(f, q, K_{\alpha}\right) \text {. }
$$

7. Domain of the multifractal spectrum of local entropies. In this section we will first establish, in a series of inequalities, an upper estimate on the multifractal spectrum for local entropies.

For this we need the notion of the Legendre transform. Let $g: I \rightarrow \mathbb{R}$ be a function defined on some interval $I$, which may be infinite or finite. We define its Legendre transform $g^{*}$ (sometimes also called the conjugate of $g$ [21]), as a function on an interval $I^{*}$, by

$$
g^{*}(y)=\inf _{x \in I}(x y+g(x)),
$$

where $I^{*}=\left\{y: g^{*}(y)\right.$ is finite $\}$. The interval $I^{*}$ is called the domain of the Legendre transform $g^{*}$.

It is clear that $g^{*}$ is a concave function:

$$
g^{*}\left(\lambda y_{1}+(1-\lambda) y_{2}\right) \geq \lambda g^{*}\left(y_{1}\right)+(1-\lambda) g^{*}\left(y_{2}\right), \quad \lambda \in[0,1], y_{1}, y_{2} \in I^{*} .
$$

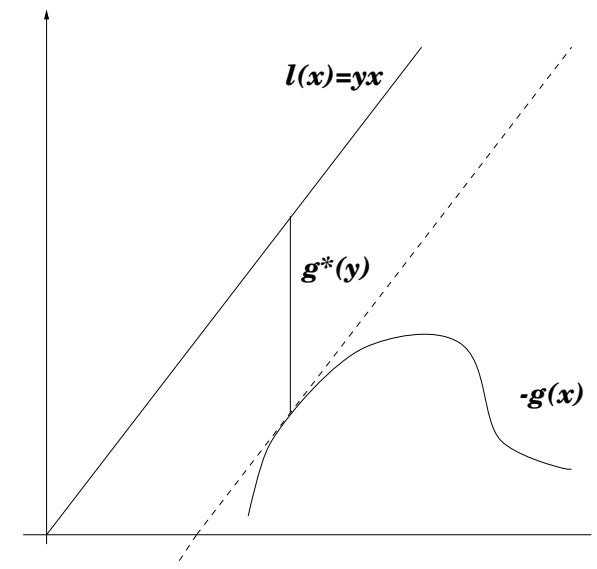

Fig. 1. Legendre transform: $g^{*}(y)=\inf _{x}(y x+g(x))=\inf _{x}(y x-(-g(x))$

Let us now relate the multifractal spectrum $\mathcal{E}(\alpha)$ to the Legendre transform of some function. In the previous sections we have shown that for any $\alpha$ and $q$ one has

$$
h_{\mathrm{top}}\left(f, K_{\alpha}\right)=q \alpha+h_{\mu}\left(f, q, K_{\alpha}\right) .
$$

We have not gained much new information about the multifractal spectrum of local entropies since we are still not able to compute $h_{\mu}\left(f, q, K_{\alpha}\right)$.

Since $K_{\alpha} \subseteq X$, using the properties of $(q, \mu)$-entropy we conclude that $h_{\mu}\left(f, q, K_{\alpha}\right) \leq h_{\mu}(f, q, X)$ for any $q \in \mathbb{R}$. We introduce the following nota- 
tion:

$$
h(q)=h_{\mu}(f, q, X) .
$$

Therefore, substituting $h(q)$ into (7.1) we conclude that

$$
h_{\text {top }}\left(f, K_{\alpha}\right) \leq q \alpha+h(q) \quad \text { for any } \alpha, q .
$$

Hence, we immediately obtain

$$
\mathcal{E}(\alpha):=h_{\text {top }}\left(f, K_{\alpha}\right) \leq h^{*}(\alpha):=\inf _{q}(q \alpha+h(q)) .
$$

In (7.2) we have deliberately made the estimate of $h_{\text {top }}\left(f, K_{\alpha}\right)$ weaker, in order to relate it to the Legendre transform in (7.3). In the next sections we will relate $h(q)$ to some other characteristics of a dynamical system, namely, the spectrum of correlation entropies.

Later, we will show that in the multifractal analysis of Gibbs measures one actually has equality in (7.3). This is due to the fact that for any $\alpha$ such that $K_{\alpha} \neq \emptyset$ there exists a unique $q=q(\alpha)$ such that

$$
h_{\mu}\left(f, q, K_{\alpha}\right)=h_{\mu}(f, q, X) .
$$

Hence, though we replaced the exact equality (7.1) by the inequality $h_{\text {top }}\left(f, K_{\alpha}\right) \leq q \alpha+h(q)$, after taking the infimum over $q$ on the right hand side we will obtain the equality

$$
h_{\text {top }}\left(f, K_{\alpha}\right)=h^{*}(\alpha) \text {. }
$$

One has to stress that this is a rather special case, and, in general, one should not expect equality between the multifractal spectrum $h_{\text {top }}\left(f, K_{\alpha}\right)$ and the Legendre transform $h^{*}(\alpha)$.

In the present section we also study the domain of $h^{*}(\alpha)$ and show that it contains the domain of the multifractal spectrum of local entropies.

We introduce the following quantities:

$$
\underline{\alpha}=\sup _{q>0}-\frac{h(q)}{q}, \quad \bar{\alpha}=\inf _{q<0}-\frac{h(q)}{q} .
$$

Obviously, $h^{*}(\alpha)=\inf _{q}(q \alpha+h(q))>-\infty$ for $\alpha \in(\underline{\alpha}, \bar{\alpha})$. The next lemma shows that the interval $[\underline{\alpha}, \bar{\alpha}]$ contains the domain of the multifractal spectrum, i.e., $K_{\alpha}=\emptyset$ (and hence $\left.\left.h_{\text {top }}\left(f, K_{\alpha}\right)=-\infty\right)\right)$ if $\alpha \notin[\underline{\alpha}, \bar{\alpha}]$.

LEMMA 7.1. The following holds:

$$
\underline{\alpha} \leq \inf _{x \in X} \bar{h}_{\mu}(f, x) \leq \sup _{x} \underline{h}_{\mu}(f, x) \leq \bar{\alpha} .
$$

Hence, $K_{\alpha}=\emptyset$ for $\alpha \notin[\underline{\alpha}, \bar{\alpha}]$.

Pr o of. Since there exist points $x \in X$ such that $\underline{h}_{\mu}(f, x)=\bar{h}_{\mu}(f, x)$ the middle inequality is obvious. 
Suppose now that $\underline{\alpha}>\inf _{x \in X} \bar{h}_{\mu}(f, x)$. This means that there exist $q_{0}>0$, $x \in X$ and $\delta>0$ such that

$$
-\frac{h\left(q_{0}\right)}{q_{0}}>\bar{h}_{\mu}(f, x)+\delta .
$$

Hence $t_{0}:=-q_{0}\left(\bar{h}_{\mu}(f, x)+\delta\right)>h(q)$. Moreover, there exist $\varepsilon>0$ and $N \in \mathbb{N}$ such that for any $n>N$ one has

$$
-\frac{1}{n} \log \mu\left(\mathcal{B}_{n}(x, \varepsilon)\right) \leq \bar{h}_{\mu}(f, x)+\delta .
$$

This implies that

$$
\mu\left(\mathcal{B}_{n}(x, \varepsilon)\right)^{q_{0}} \exp \left(-n t_{0}\right) \geq \exp \left(-n q_{0}\left(\bar{h}_{\mu}(f, x)+\delta\right)-n t_{0}\right)=1
$$

for $n>N$, where the last equality is due to the choice of $t_{0}$. Therefore

$$
M_{\mu}\left(\{x\}, q_{0}, t_{0}, \varepsilon\right)=\lim _{N \rightarrow \infty} \inf _{n \geq N} \mu\left(\mathcal{B}_{n}(x, \varepsilon)\right)^{q_{0}} \exp \left(-n t_{0}\right) \geq 1 .
$$

Hence

$$
h_{\mu}\left(T, q_{0},\{x\}\right) \geq t_{0}>h(q)=h_{\mu}\left(T, q_{0}, X\right)
$$

which is impossible due to the set monotonicity of $h_{\mu}(T, q, \cdot)$. Thus, we conclude that $\underline{\alpha} \leq \inf _{x \in X} \bar{h}_{\mu}(f, x)$.

Suppose that $\bar{\alpha}<\sup _{x \in X} \underline{h}_{\mu}(f, x)$. Then there exist $q_{0}<0, x \in X$ and $\delta>0$ such that

$$
-\frac{h\left(q_{0}\right)}{q_{0}}<\underline{h}_{\mu}(f, x)-\delta .
$$

Thus, $t_{0}:=-q_{0}\left(\underline{h}_{\mu}(f, x)-\delta\right)>h(q)$. Since

$$
\underline{h}_{\mu}(f, x)=\lim _{\varepsilon \rightarrow 0} \underline{\lim }_{n \rightarrow \infty}-\frac{1}{n} \log \mu\left(\mathcal{B}_{n}(x, \varepsilon)\right),
$$

there exist $\varepsilon>0$ and $N$ such that for any $n>N$,

$$
-\frac{1}{n} \log \mu\left(\mathcal{B}_{n}(x, \varepsilon)\right) \geq \underline{h}_{\mu}(f, x)-\delta .
$$

Thus, for any $n>N$ (note that $q_{0}<0$ ),

$$
\mu\left(\mathcal{B}_{n}(x, \varepsilon)\right)^{q_{0}} \exp \left(-n t_{0}\right) \geq \exp \left(-n q_{0}\left(\underline{h}_{\mu}(f, x)-\delta\right)-n t_{0}\right)=1,
$$

due to the choice of $t_{0}$. As in the previous case, we conclude that $h\left(f, q_{0},\{x\}\right)$ $\geq t_{0}>h\left(q_{0}\right)=h_{\mu}\left(T, q_{0}, X\right)$, contradicting the monotonicity of $h(T, q, \cdot)$. Hence $\sup _{x} \underline{h}_{\mu}(f, x) \leq \bar{\alpha}$.

REMARK. To be absolutely rigorous, we have to stress that we actually proved the following fact:

$$
(\underline{\alpha}, \bar{\alpha}) \subseteq \operatorname{dom}\left(h^{*}(\alpha)\right) \quad \text { and } \quad \operatorname{dom}(\mathcal{E}(\alpha)) \subseteq[\underline{\alpha}, \bar{\alpha}] .
$$

Therefore, we cannot deduce that $\operatorname{dom}(\mathcal{E}(\alpha)) \subseteq \operatorname{dom}\left(h^{*}(\alpha)\right)$. However, the difference $\operatorname{dom}(\mathcal{E}(\alpha)) \backslash \operatorname{dom}\left(h^{*}(\alpha)\right)$ contains at most 2 points and is irrelevant 
from the multifractal point of view. More on the situation at the end points can be found in $[22]$.

REMARK. How far the estimates $\underline{\alpha}, \bar{\alpha}$ on the domain of the multifractal spectrum are from being exact? In the later examples (Gibbs measures), where a complete description of the multifractal spectrum is known, we will see that the above estimates are sharp, meaning that not only $K_{\alpha}=\emptyset$ for $\alpha \notin[\underline{\alpha}, \bar{\alpha}]$, but $K_{\alpha} \neq \emptyset$ for $\alpha \in(\underline{\alpha}, \bar{\alpha})$.

We summarize the above results in the following statement.

THEOREM 7.2. Let $f$ be a continuous transformation of a compact metric space $(X, d)$ with finite topological entropy. Consider an invariant non-atomic Borel measure $\mu$. Then there exist $\underline{\alpha}, \bar{\alpha}$ such that:

(i) $K_{\alpha}=\emptyset$ for $\alpha \notin[\underline{\alpha}, \bar{\alpha}]$;

(ii) for $\alpha \in(\underline{\alpha}, \bar{\alpha})$ one has

$$
\mathcal{E}(\alpha)=h_{\text {top }}\left(f, K_{\alpha}\right) \leq \inf _{q}(q \alpha+h(q))=h^{*}(\alpha)
$$

where $h(q)=h_{\mu}(f, q, X)$.

8. Lower and upper $(q, \mu)$-entropy capacities. The definition of Hausdorff dimension involves coverings by balls of radius at most $\varepsilon$. If we consider coverings by balls of radius $\varepsilon$ alone we will get the notion of lower and upper capacities. The same idea can be applied to the case of the $(q, \mu)$-entropy $h_{\mu}(T, q, Z)$. Here, for the set $\mathcal{B}_{n_{i}}\left(x_{i}, \varepsilon\right)$ the role of the diameter is played by $\exp \left(-n_{i}\right)$. Thus, putting $n_{i} \equiv n$ will be equivalent to fixing the diameters of the covering sets.

Now we give a precise definition of the lower and upper $(q, \mu)$-entropy capacities. We keep the definition of the $(q, t)$-free energy $F_{\mu}(q, t, \mathcal{G})$ without any changes. However, for any $Z \subseteq X, Z \neq \emptyset$, we define

$$
C M_{\mu}^{c}(Z, q, t, \varepsilon, n)=\inf _{\mathcal{G}} F_{\mu}(q, t, \mathcal{G}),
$$

where the infimum is taken over all at most countable collections $\mathcal{G}=$ $\left\{\mathcal{B}_{n}\left(x_{i}, \varepsilon\right)\right\}_{i}$ with centers $x_{i} \in Z$, which cover $Z$. Note that $n$ is the same for all balls in $\mathcal{G}$. Hence the quantities $C M_{\mu}^{c}(Z, q, t, \varepsilon, n)$ are not necessarily monotonic in $n$. Therefore, define

$$
\begin{aligned}
& {\underline{C M_{\mu}^{c}}}_{\mu}(Z, q, t, \varepsilon)=\varliminf_{n \rightarrow \infty} C M_{\mu}^{c}(Z, q, t, \varepsilon, n), \\
& \overline{C M}_{\mu}^{c}(Z, q, t, \varepsilon)=\varlimsup_{n \rightarrow \infty} C M_{\mu}^{c}(Z, q, t, \varepsilon, n) .
\end{aligned}
$$

Again, since we consider only centered coverings, we achieve set monotonicity by putting 


$$
\begin{aligned}
& \underline{C M}_{\mu}(Z, q, t, \varepsilon)=\sup _{Z^{\prime} \subseteq Z} \frac{C M_{\mu}^{c}\left(Z^{\prime}, q, t, \varepsilon\right),}{\overline{C M}_{\mu}(Z, q, t, \varepsilon)}=\sup _{Z^{\prime} \subseteq Z} \overline{C M_{\mu}^{c}}\left(Z^{\prime}, q, t, \varepsilon\right) .
\end{aligned}
$$

There exist unique values $\underline{C h}_{\mu}(f, Z, q, \varepsilon), \overline{C h}_{\mu}(f, Z, q, \varepsilon) \in[-\infty,+\infty]$ such that

$$
\begin{aligned}
& \underline{C M}_{\mu}(Z, q, t, \varepsilon)= \begin{cases}\infty & \text { if } t<\overline{C h}_{\mu}(f, Z, q, \varepsilon), \\
0 & \text { if } t>\overline{C h}_{\mu}(f, Z, q, \varepsilon),\end{cases} \\
& \overline{C M}_{\mu}(Z, q, t, \varepsilon)= \begin{cases}\infty & \text { if } t<\overline{C h}_{\mu}(f, Z, q, \varepsilon), \\
0 & \text { if } t>\overline{C h}_{\mu}(f, Z, q, \varepsilon) .\end{cases}
\end{aligned}
$$

Finally, we define the lower and upper entropy capacities as

$$
\underline{C h}_{\mu}(f, Z, q)=\varlimsup_{\varepsilon \rightarrow 0} \underline{C h}_{\mu}(f, Z, q, \varepsilon), \quad \overline{C h}_{\mu}(f, Z, q)=\varlimsup_{\varepsilon \rightarrow 0} \overline{C h}_{\mu}(f, Z, q, \varepsilon) .
$$

The lower and upper capacities admit a different equivalent definition. Namely, for every set $Z \subseteq X, Z \neq \emptyset$, define

$$
\Lambda(Z, q, \varepsilon, n)=\inf _{\mathcal{G}} \sum_{i} \mu\left(\mathcal{B}_{n}\left(x_{i}, \varepsilon\right)\right)^{q},
$$

where the infimum is taken over all finite or countable collections $\mathcal{G}=$ $\left\{\mathcal{B}_{n}\left(x_{i}, \varepsilon\right)\right\}_{i}$ with $x_{i} \in Z$ and such that $Z \subseteq \bigcup_{i} \mathcal{B}_{n}\left(x_{i}, \varepsilon\right)$. By definition we let $\Lambda(\emptyset, q, \varepsilon, n)=0$.

The next statement (similar to [17, Theorem 2.2]) gives an equivalent definition of $\underline{C h}_{\mu}(f, Z, q, \varepsilon)$ and $\overline{C h}_{\mu}(f, Z, q, \varepsilon)$.

Lemma 8.1. For any $Z \subseteq X, Z \neq \emptyset$, we have

$$
\begin{aligned}
& \underline{C h}_{\mu}(f, Z, q, \varepsilon)=\sup _{Z^{\prime} \subseteq Z} \varliminf_{n \rightarrow \infty} \frac{\log \Lambda\left(Z^{\prime}, q, \varepsilon, n\right)}{n}, \\
& \overline{C h}_{\mu}(f, Z, q, \varepsilon)=\sup _{Z^{\prime} \subseteq Z} \varlimsup_{n \rightarrow \infty} \frac{\log \Lambda\left(Z^{\prime}, q, \varepsilon, n\right)}{n} .
\end{aligned}
$$

Pr o of. We prove the equality only for the case of lower entropy capacity. The other case is analogous. Define for simplicity

$$
a=\underline{C h}_{\mu}(Z, q, \varepsilon), \quad b=\sup _{Z^{\prime} \subseteq Z} \varliminf_{n \rightarrow \infty} \frac{\Lambda\left(Z^{\prime}, q, \varepsilon, n\right)}{n} .
$$

Let $\delta>0$. Then by definition (8.2), for every $Z^{\prime} \subseteq Z$ one has

$$
0=\underline{C M} \mu\left(Z^{\prime}, q, a+\delta, \varepsilon\right)=\varliminf_{n \rightarrow \infty} \inf _{\mathcal{G}} \sum_{i} \mu\left(\mathcal{B}_{n}\left(x_{i}, \varepsilon\right)\right)^{q} \exp (-n(a+\delta)) .
$$

Therefore there exists a sequence $\left\{n_{k}\right\}, n_{k} \rightarrow \infty$ as $k \rightarrow \infty$, and a sequence of $Z^{\prime}$-centered coverings $\mathcal{G}_{k}=\left\{\mathcal{B}_{n_{k}}\left(x_{i}, \varepsilon\right)\right\}$ such that $F_{\mu}\left(q, a+\delta, \mathcal{G}_{n_{k}}\right) \rightarrow 0$ as $k \rightarrow \infty$. Since $\Lambda\left(Z^{\prime}, q, \varepsilon, n_{k}\right) \exp \left(-n_{k}(a+\delta)\right) \leq F_{\mu}\left(q, a+\delta, \mathcal{G}_{n_{k}}\right)$ we conclude 
that

$$
\varliminf_{n \rightarrow \infty} \frac{\log \Lambda\left(Z^{\prime}, q, \varepsilon, n\right)}{n} \leq a+\delta .
$$

Since $Z^{\prime}$ is an arbitrary subset of $Z$ and $\delta>0$ is arbitrary, we conclude that $b \leq a$.

Now we prove the opposite inequality. For any $\delta>0$ one has

$$
\underline{C M}_{\mu}(Z, q, a-\delta, \varepsilon)=\sup _{Z^{\prime} \subseteq Z} \underline{C M}_{\mu}^{c}\left(Z^{\prime}, q, a-\delta, \varepsilon\right)=\infty .
$$

Hence there exists a set $Z^{\prime} \subseteq Z$ such that

$$
\underline{C M}_{\mu}^{c}\left(Z^{\prime}, q, a-\delta, \varepsilon\right)=\varliminf_{n \rightarrow \infty} \inf _{\mathcal{G}} \sum_{i} \mu\left(\mathcal{B}_{n}\left(x_{i}, \varepsilon\right)\right)^{q} \exp (-n(a-\delta))>2
$$

and therefore for sufficiently large $N$ and all $n>N$ and every centered covering $\mathcal{G}$ of $Z^{\prime}$ one has

$$
F_{\mu}(q, a-\delta, G)=\left(\sum_{i} \mu\left(\mathcal{B}_{n}\left(x_{i}, \varepsilon\right)\right)^{q}\right) \exp (-n(a-\delta))>1 .
$$

Thus, for all $n>N$,

$$
\frac{\log \Lambda\left(Z^{\prime}, q, \varepsilon, n\right)}{n} \geq a-\delta .
$$

This implies that $b \geq \underline{\lim }_{n \rightarrow \infty}\left(\log \Lambda\left(Z^{\prime}, q, \varepsilon, n\right)\right) / n \geq a-\delta$. Since $\delta$ was arbitrary we conclude that $b \geq a$.

It is obvious that the $(q, \mu)$-entropy and the lower and upper entropy capacities satisfy the inequalities

$$
h_{\mu}(f, q, Z) \leq \underline{C h}_{\mu}(f, q, Z) \leq \overline{C h}_{\mu}(f, q, Z)
$$

for any $q \in \mathbb{R}$ and all $Z \subseteq X$. Therefore we can substitute $\underline{C h}(q):=$ $\underline{C h}_{\mu}(f, q, X)$ and $\overline{C h}(q):=\overline{C h}_{\mu}(f, q, X)$ for $h(q)=h_{\mu}(f, q, X)$ in the statement of Theorem 7.2. Thus we obtain the following theorem.

THEOREM 8.2. Let $f$ be a continuous transformation of a compact metric space $(X, d)$ with finite topological entropy. Consider an invariant non-atomic Borel measure $\mu$. Then there exist $\underline{\alpha}, \bar{\alpha}$ such that

(i) $K_{\alpha}=\emptyset$ for $\alpha \notin[\underline{\alpha}, \bar{\alpha}]$;

(ii) for $\alpha \in(\underline{\alpha}, \bar{\alpha})$ one has

$$
\mathcal{E}(\alpha)=h_{\text {top }}\left(f, K_{\alpha}\right) \leq \underline{C h}^{*}(\alpha) \leq \overline{C h}^{*}(\alpha),
$$

where $\underline{C h}(q)=\underline{C h}_{\mu}(f, q, X)$ and $\overline{C h}(q)=\overline{C h}_{\mu}(f, q, X)$.

9. Correlation entropies. The spectra of generalized dimensions have been successfully used in the multifractal analysis of local dimensions [17, 16]. Two spectra have been considered: the first, named after Rényi, is based on box counting, the second (sometimes called the Hentschel-Procaccia 
spectrum) is based on correlation integrals. Under mild assumptions these spectra coincide $[9,17]$.

Generalizations to the entropy case were made in $[8,3]$. However, the first approach produces the Rényi entropies, which do not contain any essential information about the dynamical system [23].

On the other hand, the correlation entropies, which we define in this section, do not present such problems and suit the purposes of the multifractal analysis of local entropies quite well.

For a given $q \neq 1$ define

$$
I(q, \varepsilon, n)=\int \mu\left(\mathcal{B}_{n}(x, \varepsilon)\right)^{q-1} d \mu .
$$

The lower and upper correlation entropies are then defined as

$$
\begin{aligned}
& \underline{H}_{\mu}(f, q)=\lim _{\varepsilon \rightarrow 0} \varliminf_{n \rightarrow \infty}-\frac{1}{(q-1) n} \log I(q, \varepsilon, n), \\
& \bar{H}_{\mu}(f, q)=\lim _{\varepsilon \rightarrow 0} \varlimsup_{n \rightarrow \infty}-\frac{1}{(q-1) n} \log I(q, \varepsilon, n) .
\end{aligned}
$$

We define the spectrum of correlation entropies at $q=1$ by continuity in $q$ :

$$
I(1, \varepsilon, n)=\int \log \mu\left(\mathcal{B}_{n}(x, \varepsilon)\right) d \mu,
$$

and

$$
\underline{H}_{\mu}(f, 1)=\lim _{\varepsilon \rightarrow 0} \varliminf_{n \rightarrow \infty}-\frac{1}{n} I(1, \varepsilon, n), \quad \bar{H}_{\mu}(f, 1)=\lim _{\varepsilon \rightarrow 0} \varlimsup_{n \rightarrow \infty}-\frac{1}{n} I(1, \varepsilon, n) .
$$

Applying the Brin-Katok formula and Fatou lemma we conclude that actually

$$
\underline{H}_{\mu}(f, 1)=\bar{H}_{\mu}(f, 1)=h_{\mu}(f) \text {. }
$$

One can easily show that $\underline{H}_{\mu}(f, q)$ and $\bar{H}_{\mu}(f, q)$ are continuous functions for $q \in[0,1)$ and $q \in(1, \infty)$, with possible singularities at $q=1$ (see the last section for examples).

Moreover, the following (quite rough) estimates are known:

$$
\begin{aligned}
0 & \leq \underline{H}_{\mu}(f, q) \leq \bar{H}_{\mu}(f, q) \leq h_{\mu}(f) & & \text { for } q>1, \\
h_{\mu}(f) & \leq \underline{H}_{\mu}(f, q) \leq \bar{H}_{\mu}(f, q) \leq h_{\mathrm{top}}(f) & & \text { for } q \in[0,1) .
\end{aligned}
$$

Since we will later use the correlation entropies in our estimates of the multifractal spectrum, which we would like to keep as sharp as possible, we will only use the lower correlation entropies. Hence, we set

$$
H_{\mu}(f, q)=\underline{H}_{\mu}(f, q) \text {. }
$$

10. Entropy doubling condition and correlation entropies. In this section we assume that the invariant measure satisfies the entropy doubling condition (see (4.3)). This will allow us to relate the multifractal spectrum 
for local entropies to the spectrum of correlation entropies, defined in the previous section.

LEMMA 10.1. If $\mu$ satisfies the entropy doubling condition then for every sufficiently small $\varepsilon>0$ and any $q>1$ there exist constants $C_{1}, C_{2}$ such that for any $Z \subseteq X$ one has

$$
C_{1} \Lambda(Z, q, 2 \varepsilon, n) \leq \int \mu\left(\mathcal{B}_{n}(x, \varepsilon)\right)^{q-1} d \mu \leq C_{2} \Lambda(X, q, \varepsilon, n) .
$$

Proof. Let $Z \subseteq X$ and $E=\left\{x_{i}\right\}$ be some maximal $(n, \varepsilon)$-separated set, i.e. for every $i \neq j$ one has $d_{n}\left(x_{i}, x_{j}\right)>\varepsilon$ and one cannot add any other points to $E$ without breaking the separation condition. This in particular implies that $\mathcal{B}_{n}\left(x_{i}, \varepsilon / 2\right) \cap \mathcal{B}_{n}\left(x_{j}, \varepsilon / 2\right)=\emptyset$ and $X \subseteq \bigcup_{i} \mathcal{B}_{n}\left(x_{i}, \varepsilon\right)$.

Obviously, for $q>1$,

$$
\begin{aligned}
\int \mu\left(\mathcal{B}_{n}(x, \varepsilon)\right)^{q-1} d \mu & \geq \sum_{i} \int_{\mathcal{B}_{n}\left(x_{i}, \varepsilon / 2\right)} \mu\left(\mathcal{B}_{n}(x, \varepsilon)\right)^{q-1} d \mu \\
& \geq \sum_{i} \mu\left(\mathcal{B}_{n}\left(x_{i}, \varepsilon / 2\right)\right)^{q},
\end{aligned}
$$

since $\mathcal{B}_{n}\left(x_{i}, \varepsilon / 2\right) \subseteq \mathcal{B}_{n}(x, \varepsilon)$ for every $x \in \mathcal{B}_{n}\left(x_{i}, \varepsilon / 2\right)$.

On the other hand, for any $i$ such that $\mathcal{B}_{n}\left(x_{i}, \varepsilon\right) \cap Z \neq \emptyset$ choose some $z_{i} \in$ $\mathcal{B}_{n}\left(x_{i}, \varepsilon\right) \cap Z$. Denote the set of all such $i$ 's by $I^{\prime}$. Thus $\mathcal{B}_{n}\left(x_{i}, \varepsilon\right) \subseteq \mathcal{B}_{n}\left(z_{i}, 2 \varepsilon\right)$ and $Z \subseteq \bigcup_{i \in I^{\prime}} \mathcal{B}_{n}\left(z_{i}, 2 \varepsilon\right)$. Hence, $\left\{\mathcal{B}_{n}\left(z_{i}, 2 \varepsilon\right)\right\}_{i \in I^{\prime}}$ is a centered covering of $Z$. Note that $\mathcal{B}_{n}\left(z_{i}, \varepsilon\right) \subseteq \mathcal{B}_{n}\left(x_{i}, 2 \varepsilon\right)$ as well.

Let $C=C(\varepsilon) \in(1, \infty)$ be such that

$$
\sup _{x} \frac{\mu\left(\mathcal{B}_{n}(x, 2 \varepsilon)\right)}{\mu\left(\mathcal{B}_{n}(x, \varepsilon)\right)}<C \quad \text { and } \quad \sup _{x} \frac{\mu\left(\mathcal{B}_{n}(x, \varepsilon)\right)}{\mu\left(\mathcal{B}_{n}(x, \varepsilon / 2)\right)}<C
$$

for all $n$. Such a $C$ exists due to the entropy doubling condition. Therefore,

$$
\begin{aligned}
& \Lambda(Z, q, 2 \varepsilon, n) \leq \sum_{i \in I^{\prime}} \mu\left(\mathcal{B}_{n}\left(z_{i}, 2 \varepsilon\right)\right)^{q} \leq C^{q} \sum_{i \in I^{\prime}} \mu\left(\mathcal{B}_{n}\left(z_{i}, \varepsilon\right)\right)^{q} \\
& \quad \leq C^{q} \sum_{i \in I^{\prime}} \mu\left(\mathcal{B}_{n}\left(x_{i}, 2 \varepsilon\right)\right)^{q} \leq C^{2 q} \sum_{i \in I} \mu\left(\mathcal{B}_{n}\left(x_{i}, \varepsilon\right)\right)^{q} \leq C^{3 q} \sum_{i \in I} \mu\left(\mathcal{B}_{n}\left(x_{i}, \varepsilon / 2\right)\right)^{q} .
\end{aligned}
$$

Therefore, comparing (10.1) with the previous estimate we conclude that for $q>1$ and any $Z \subseteq X$,

$$
\frac{1}{C^{3 q}} \Lambda(Z, q, 2 \varepsilon, n) \leq \int \mu\left(\mathcal{B}_{n}(x, \varepsilon)\right)^{q-1} d \mu .
$$

Let $E=\left\{x_{i}\right\}$ be an arbitrary $(n, \varepsilon)$-generating set, i.e. $X=\bigcup_{i} \mathcal{B}_{n}\left(x_{i}, \varepsilon\right)$. Choose $C>1$ such that

$$
\sup _{x} \frac{\mu\left(\mathcal{B}_{n}(x, 2 \varepsilon)\right)}{\mu\left(\mathcal{B}_{n}(x, \varepsilon)\right)}<C
$$


for all $n$. Then

$$
\begin{aligned}
\int \mu\left(\mathcal{B}_{n}(x, \varepsilon)\right)^{q-1} d \mu & \leq \sum_{i} \int_{\mathcal{B}_{n}\left(x_{i}, \varepsilon\right)} \mu\left(\mathcal{B}_{n}(x, \varepsilon)\right)^{q-1} d \mu \\
& \leq \sum_{i} \mu\left(\mathcal{B}_{n}\left(x_{i}, 2 \varepsilon\right)\right)^{q-1} \mu\left(\mathcal{B}_{n}\left(x_{i}, \varepsilon\right)\right) \\
& \leq C^{q-1} \sum_{i} \mu\left(\mathcal{B}_{n}\left(x_{i}, \varepsilon\right)\right)^{q} .
\end{aligned}
$$

Therefore, since $E$ is an arbitrary $(n, \varepsilon)$-generating set we conclude that for $q>1$,

$$
\int \mu\left(\mathcal{B}_{n}(x, \varepsilon)\right)^{q-1} d \mu \leq C^{q-1} \Lambda(X, q, \varepsilon, n) .
$$

LEMma 10.2. If $\mu$ satisfies the entropy doubling condition then for every sufficiently small $\varepsilon>0$ and any $q<1$ there exist constants $D_{1}$ such that for any $Z \subseteq X$ one has

$$
D_{1} \Lambda(Z, q, \varepsilon, n) \leq \int \mu\left(\mathcal{B}_{n}(x, \varepsilon)\right)^{q-1} d \mu \leq \Lambda(X, q, \varepsilon / 2, n) .
$$

Proof. We start with the second inequality. Let $E=\left\{x_{i}\right\}$ be an arbitrary $(n, \varepsilon / 2)$-generating set. Then

$$
\begin{aligned}
\int \mu\left(\mathcal{B}_{n}(x, \varepsilon)\right)^{q-1} d \mu & \leq \sum_{i} \int_{\mathcal{B}_{n}\left(x_{i}, \varepsilon / 2\right)} \mu\left(\mathcal{B}_{n}(x, \varepsilon)\right)^{q-1} d \mu \\
& \leq \sum_{i} \mu\left(\mathcal{B}_{n}\left(x_{i}, \varepsilon / 2\right)\right)^{q},
\end{aligned}
$$

since if $x \in \mathcal{B}_{n}\left(x_{i}, \varepsilon / 2\right)$ then $\mathcal{B}_{n}\left(x_{i}, \varepsilon / 2\right) \subseteq \mathcal{B}_{n}(x, \varepsilon)$. Thus

$$
\int \mu\left(\mathcal{B}_{n}(x, \varepsilon)\right)^{q-1} d \mu \leq \Lambda(X, q, \varepsilon / 2, n) .
$$

Let $E=\left\{x_{i}\right\}$ be an arbitrary maximal $(n, 2 \varepsilon)$-separated set. Thus $\mathcal{B}_{n}\left(x_{i}, \varepsilon\right) \cap \mathcal{B}_{n}\left(x_{j}, \varepsilon\right)=\emptyset$ for $i \neq j$ and

$$
\begin{aligned}
\int \mu\left(\mathcal{B}_{n}(x, \varepsilon)\right)^{q-1} d \mu & \geq \sum_{i} \int_{\mathcal{B}_{n}\left(x_{i}, \varepsilon\right)} \mu\left(\mathcal{B}_{n}(x, \varepsilon)\right)^{q-1} d \mu \\
& \geq \sum_{i} \mu\left(\mathcal{B}_{n}\left(x_{i}, 2 \varepsilon\right)\right)^{q-1} \mu\left(\mathcal{B}_{n}\left(x_{i}, \varepsilon\right)\right) \\
& \geq \frac{1}{C} \sum_{i} \mu\left(\mathcal{B}_{n}\left(x_{i}, 2 \varepsilon\right)\right)^{q},
\end{aligned}
$$

where we have used the fact that for $x \in \mathcal{B}_{n}\left(x_{i}, \varepsilon\right)$ one has $\mathcal{B}_{n}(x, \varepsilon) \subseteq$ $\mathcal{B}_{n}\left(x_{i}, 2 \varepsilon\right)$, and $C>1$ is such that

$$
\sup _{x} \frac{\mu\left(\mathcal{B}_{n}(x, 2 \varepsilon)\right)}{\mu\left(\mathcal{B}_{n}(x, \varepsilon)\right)}<C .
$$


Let $Z \subseteq X$ and $\mathcal{G}=\left\{\mathcal{B}_{n}\left(z_{j}, \varepsilon\right)\right\}$ be an arbitrary centered covering of $Z$. Let $C$ be such that

$$
\sup _{x} \frac{\mu\left(\mathcal{B}_{n}(x, 4 \varepsilon)\right)}{\mu\left(\mathcal{B}_{n}(x, 2 \varepsilon)\right)}<C \quad \text { and } \quad \sup _{x} \frac{\mu\left(\mathcal{B}_{n}(x, 2 \varepsilon)\right)}{\mu\left(\mathcal{B}_{n}(x, \varepsilon)\right)}<C
$$

for all $n \geq 1$. For any $j$ there exists some $i=i(j)$ such that $z_{j} \in \mathcal{B}_{n}\left(x_{i}, 2 \varepsilon\right)$. Denote the set of those $i$ by $I^{\prime}$. First, consider $q \in[0,1)$. Since $\mathcal{B}_{n}\left(z_{j}, \varepsilon\right) \subseteq$ $\mathcal{B}_{n}\left(x_{i}, 4 \varepsilon\right)$ we conclude that

$$
\sum_{j} \mu\left(\mathcal{B}_{n}\left(z_{j}, \varepsilon\right)\right)^{q} \leq \sum_{i \in I^{\prime}} \mu\left(\mathcal{B}_{n}\left(x_{i}, 4 \varepsilon\right)\right)^{q} \leq C^{q} \sum_{i \in I^{\prime}} \mu\left(\mathcal{B}_{n}\left(x_{i}, 2 \varepsilon\right)\right)^{q} .
$$

Consider now $q<0$. If $z_{j} \in \mathcal{B}_{n}\left(x_{i}, 2 \varepsilon\right)$ then $\mathcal{B}_{n}\left(x_{i}, 2 \varepsilon\right) \subseteq \mathcal{B}_{n}\left(z_{j}, 4 \varepsilon\right)$. Therefore

$$
\sum_{i \in I^{\prime}} \mu\left(\mathcal{B}_{n}\left(x_{i}, 2 \varepsilon\right)\right)^{q} \geq \sum_{j} \mu\left(\mathcal{B}_{n}\left(z_{j}, 4 \varepsilon\right)\right)^{q} \geq C^{2 q} \sum_{j} \mu\left(\mathcal{B}_{n}\left(z_{j}, \varepsilon\right)\right)^{q} .
$$

In both cases, there exists some constant $D$ such that

$$
\sum_{i \in I^{\prime}} \mu\left(\mathcal{B}_{n}\left(x_{i}, 2 \varepsilon\right)\right)^{q} \geq D \sum_{j} \mu\left(\mathcal{B}_{n}\left(z_{j}, \varepsilon\right)\right)^{q} \geq D \Lambda(Z, q, \varepsilon, n) .
$$

Comparing this to $(10.2)$ we conclude that there exists $D_{1}$ such that for any $Z \subseteq X$

$$
\int \mu\left(\mathcal{B}_{n}(x, \varepsilon)\right)^{q-1} d \mu \geq D_{1} \Lambda(Z, q, \varepsilon, n)
$$

As a direct consequence of the above estimates we obtain the following result.

Lemma 10.3. Suppose $\mu$ satisfies the entropy doubling condition. Then for $q<1$ one has

$$
\overline{C h}_{\mu}(f, q, X, 2 \varepsilon) \leq \varlimsup_{n \rightarrow \infty} \frac{1}{n} \log \int \mu\left(\mathcal{B}_{n}(x, \varepsilon)\right)^{q-1} d \mu \leq \overline{C h}_{\mu}(f, q, X, \varepsilon),
$$

and for $q>1$,

$$
\underline{C h}_{\mu}(f, q, X, 2 \varepsilon) \leq \underline{\lim }_{n \rightarrow \infty} \frac{1}{n} \log \int \mu\left(\mathcal{B}_{n}(x, \varepsilon)\right)^{q-1} d \mu \leq \underline{C h}_{\mu}(f, q, X, \varepsilon) .
$$

Therefore, for $q<1$ we have

$$
\begin{aligned}
H_{\mu}(f, q) & =\lim _{\varepsilon \rightarrow 0} \frac{\lim _{n \rightarrow \infty}}{n \rightarrow \infty} \frac{1}{(q-1) n} \log \int \mu\left(\mathcal{B}_{n}(x, \varepsilon)\right)^{q-1} d \mu \\
& =-\frac{1}{q-1} \underline{C h}_{\mu}(f, q, X),
\end{aligned}
$$

and for $q>1$,

$$
\begin{aligned}
H_{\mu}(f, q) & =\lim _{\varepsilon \rightarrow 0} \frac{\lim _{n \rightarrow \infty}}{n \rightarrow} \frac{1}{(q-1) n} \log \int \mu\left(\mathcal{B}_{n}(x, \varepsilon)\right)^{q-1} d \mu \\
& =-\frac{1}{q-1} \overline{C h}_{\mu}(f, q, X) .
\end{aligned}
$$


In both cases, one has $(1-q) H_{\mu}(f, q) \geq h_{\mu}(f, q, X)$, and hence we easily obtain a third version of our main result on the multifractal analysis of local entropies.

THEOREM 10.4. Let $f$ be a continuous transformation of a compact metric space $(X, d)$ with finite topological entropy. Consider an invariant non-atomic Borel measure $\mu$ which satisfies the entropy doubling condition. Then there exist $\underline{\alpha}, \bar{\alpha}$ such that:

(i) $K_{\alpha}=\emptyset$ for $\alpha \notin[\underline{\alpha}, \bar{\alpha}]$;

(ii) for $\alpha \in(\underline{\alpha}, \bar{\alpha})$ one has

$$
\mathcal{E}(\alpha)=h_{\text {top }}\left(f, K_{\alpha}\right) \leq \inf _{q}(q \alpha+T(q))=T^{*}(\alpha),
$$

where $T(q)=(1-q) H_{\mu}(f, q)$.

Now we define those systems $(X, \mu, f)$ for which the multifractal formalism is valid.

DEFINITION 10.5. We say that the multifractal formalism for local entropies is valid for the dynamical system $(X, \mu, f)$ if there exist $\underline{\alpha}, \bar{\alpha}$ such that:

(i) $K_{\alpha}=\emptyset$ for $\alpha \notin[\underline{\alpha}, \bar{\alpha}]$ and $K_{\alpha} \neq \emptyset$ for $\alpha \in(\underline{\alpha}, \bar{\alpha})$; $(\underline{\alpha}, \bar{\alpha})$;

(ii) $\mathcal{E}(\alpha)=h_{\text {top }}\left(f, K_{\alpha}\right)$ is a smooth convex function of $\alpha$ on the interval

(iii) let $T(q)=(1-q) H_{\mu}(f, q)$; then

$$
\mathcal{E}(\alpha)=\inf _{q}(q \alpha+T(q))=T^{*}(\alpha)
$$

for all $\alpha \in(\underline{\alpha}, \bar{\alpha})$.

11. Discussion of another entropy characteristic. The definition of $(q, \mu)$-entropy involves coverings with centers in a given set. This suits our technical purposes. One could argue, however, how natural this definition is.

The following definition, in our opinion, is closer in spirit to the definition of Hausdorff dimension (or topological entropy for a non-compact or non-invariant set).

Again, let $Z \subseteq X, Z \neq \emptyset$, and $q, t \in \mathbb{R}$. We define

$$
m_{\mu}(Z, q, t, \varepsilon, n)=\inf _{\mathcal{G}} \sum_{i} \mu\left(\mathcal{B}_{n_{i}}\left(x_{i}, \varepsilon\right)\right)^{q} \exp \left(-t n_{i}\right),
$$

where the infimum is taken over all collections $\mathcal{G}=\left\{\mathcal{B}_{n_{i}}\left(x_{i}, \varepsilon\right)\right\}$ with $n_{i} \geq n$ covering $Z$, i.e. $Z \subseteq \bigcup_{i} \mathcal{B}_{n_{i}}\left(x_{i}, \varepsilon\right)$. Note that we do not require the centers $x_{i}$ to be in $Z$. Thus the quantities $m_{\mu}(Z, q, t, \varepsilon, n)$ are monotonic with respect 
to the first argument. Moreover,

$$
m_{\mu}(Z, q, t, \varepsilon, n) \leq M_{\mu}^{c}(Z, q, t, \varepsilon, n),
$$

since we have only enlarged the class of admissible coverings and therefore the infimum cannot be larger.

Obviously, $m_{\mu}(Z, q, t, \varepsilon, n)$ is non-decreasing in $n$, therefore the following limit exists:

$$
m_{\mu}(Z, q, t, \varepsilon)=\lim _{n \rightarrow \infty} m_{\mu}(Z, q, t, \varepsilon, n)=\sup _{n \geq 1} m_{\mu}(Z, q, t, \varepsilon, n) .
$$

The qualitative behavior of $m_{\mu}(Z, q, t, \varepsilon)$ is similar to that of $M_{\mu}(Z, q, t, \varepsilon)$. Namely, there exists a critical value $h_{\mu}^{*}(f, q, Z, \varepsilon)$ such that

$$
m_{\mu}(Z, q, t, \varepsilon)= \begin{cases}+\infty, & t<h_{\mu}^{*}(f, q, Z, \varepsilon), \\ 0, & t>h_{\mu}^{*}(f, q, Z, \varepsilon) .\end{cases}
$$

Taking into account (11.1) we get

$$
m_{\mu}(Z, q, t, \varepsilon) \leq M_{\mu}^{c}(Z, q, t, \varepsilon) \leq \sup _{Z^{\prime} \subseteq Z} M_{\mu}^{c}\left(Z^{\prime}, q, t, \varepsilon\right)=M_{\mu}(Z, q, t, \varepsilon) .
$$

Therefore $h_{\mu}^{*}(f, q, Z, \varepsilon) \leq h_{\mu}(f, q, Z, \varepsilon)$, and

$$
h_{\mu}^{*}(f, q, Z):=\varlimsup_{\varepsilon \rightarrow 0} h_{\mu}^{*}(f, q, Z, \varepsilon) \leq h_{\mu}(f, q, Z) .
$$

The next lemma establishes sufficient conditions for equality of $h_{\mu}^{*}(f, q, Z)$ and $h_{\mu}(f, q, Z)$.

LEMMA 11.1. The equality $h_{\mu}^{*}(f, q, Z)=h_{\mu}(f, q, Z)$ holds for every $Z \subseteq X$ if $q \leq 0$, and also if $q>0$ and $\mu$ satisfies the entropy doubling condition.

Proof. Suppose $h_{\mu}^{*}(f, q, Z)<h_{\mu}(f, q, Z)$. Since by definition $h_{\mu}^{*}(f, q, Z)$ $=\varlimsup_{\varepsilon \rightarrow 0} h_{\mu}^{*}(f, q, Z, \varepsilon)$ and $h_{\mu}(f, q, Z)=\varlimsup_{\lim _{\varepsilon \rightarrow 0}} h_{\mu}(f, q, Z, \varepsilon)$, there exist $\varepsilon>0$ and $\delta>0$ such that

$$
h_{\mu}^{*}(f, q, Z, \varepsilon / 2)+\delta<h_{\mu}(f, q, Z, \varepsilon) .
$$

This implies that

$$
\begin{aligned}
m_{\mu}\left(Z, q, h_{\mu}^{*}(f, q, Z)+\delta, \varepsilon / 2\right) & =0, \\
M_{\mu}\left(Z, q, h_{\mu}^{*}(f, q, Z)+\delta, \varepsilon\right) & =\infty .
\end{aligned}
$$

Denote for simplicity $h_{\mu}^{*}(f, q, Z)+\delta$ by $t$ and let $C$ be any number greater than 1. From (11.2) we conclude that there exists a set $Z^{\prime} \subseteq Z$ such that $M_{\mu}^{c}\left(Z^{\prime}, q, t, \varepsilon\right)>C^{2|q|}$. Hence, there exists an integer $N$ such that

$$
F_{\mu}\left(q, t, \mathcal{G}^{\prime}\right)=\sum_{i} \mu\left(\mathcal{B}_{n_{i}}\left(z_{i}, \varepsilon\right)\right)^{q} \exp \left(-n_{i} t\right)>C^{2|q|}
$$

for any collection $\mathcal{G}^{\prime}=\left\{\mathcal{B}_{n_{i}}\left(z_{i}, \varepsilon\right)\right\}$ with $n_{i} \geq N$ and $z_{i} \in Z^{\prime}$ covering $Z^{\prime}$. 
On the other hand, since $m_{\mu}\left(Z^{\prime}, q, t, \varepsilon / 2\right)=0$, there exists a collection $\mathcal{G}^{\prime}=\left\{\mathcal{B}_{n_{i}}\left(x_{i}, \varepsilon / 2\right)\right\}$ covering $Z$ with $n_{i} \geq N$ such that

$$
F_{\mu}(q, t, \mathcal{G})=\sum_{i} \mu\left(\mathcal{B}_{n_{i}}\left(x_{i}, \varepsilon / 2\right)\right)^{q} \exp \left(-n_{i} t\right)<\frac{1}{2 C}
$$

Without loss of generality we may assume that $\mathcal{B}_{n_{i}}\left(x_{i}, \varepsilon / 2\right) \cap Z^{\prime} \neq \emptyset$. Otherwise, after deleting these sets from $\mathcal{G}$ we obtain a smaller covering with this property, which still satisfies (11.4).

For any $i$ choose some $z_{i} \in \mathcal{B}_{n_{i}}\left(x_{i}, \varepsilon / 2\right) \cap Z^{\prime}$. Obviously, $\mathcal{B}_{n_{i}}\left(x_{i}, \varepsilon / 2\right) \subseteq$ $\mathcal{B}_{n_{i}}\left(z_{i}, \varepsilon\right)$. Therefore by (11.3) we obtain

$$
F_{\mu}\left(q, t, \mathcal{G}^{\prime}\right)=\sum_{i} \mu\left(\mathcal{B}_{n_{i}}\left(z_{i}, \varepsilon\right)\right)^{q} \exp \left(-t n_{i}\right)>C^{2|q|} .
$$

Now, we have to consider two cases: $q \leq 0$ and $q>0$. If $q \leq 0$ then $\mu\left(\mathcal{B}_{n_{i}}\left(x_{i}, \varepsilon / 2\right)\right)^{q} \geq \mu\left(\mathcal{B}_{n_{i}}\left(z_{i}, \varepsilon\right)\right)^{q}$ and hence

$$
1 \leq C^{2|q|}<F_{\mu}\left(q, t, \mathcal{G}^{\prime}\right) \leq F_{\mu}(q, t, \mathcal{G})<\frac{1}{2 C}<\frac{1}{2}
$$

Thus we have arrived at a contradiction.

If $q>0$ then the previous argument does not work. However, since $\mu$ satisfies the entropy doubling condition, we can choose $C>1$ such that

$$
\sup _{x} \frac{\mu\left(\mathcal{B}_{n}(x, \varepsilon)\right)}{\mu\left(\mathcal{B}_{n}(x, \varepsilon / 2)\right)}<C
$$

for all $n$. Therefore

$$
\mu\left(\mathcal{B}_{n_{i}}\left(z_{i}, \varepsilon\right)\right) \leq C \mu\left(\mathcal{B}_{n_{i}}\left(z_{i}, \varepsilon / 2\right)\right) \leq C \mu\left(\mathcal{B}_{n_{i}}\left(x_{i}, \varepsilon\right)\right) \leq C^{2} \mu\left(\mathcal{B}_{n_{i}}\left(x_{i}, \varepsilon / 2\right)\right),
$$

since $\mathcal{B}_{n_{i}}\left(z_{i}, \varepsilon / 2\right) \subseteq \mathcal{B}_{n_{i}}\left(x_{i}, \varepsilon\right)$ if $z_{i} \in \mathcal{B}_{n_{i}}\left(x_{i}, \varepsilon / 2\right)$. The above inequality implies that for $q>0$ one has

$$
C^{2|q|}<F_{\mu}\left(q, t, \mathcal{G}^{\prime}\right) \leq C^{2 q} F_{\mu}(q, t, \mathcal{G}) \leq \frac{C^{2 q}}{2 C},
$$

and we have arrived at a contradiction again.

12. Examples. In this section we discuss three examples which illustrate the general results obtained in the previous section. We start with the following simple example.

12.1. Homogeneous measures

DEFINITION 12.1 (see [26]). Let $f$ be a continuous transformation of a compact metric space $(X, d)$. A Borel measure $\mu$ on $X$ is said to be $f$-homogeneous if for each $\varepsilon>0$ there exist $\delta>0$ and $c>0$ such that

$$
\mu\left(\mathcal{B}_{n}(y, \delta)\right) \leq c \mu\left(\mathcal{B}_{n}(x, \varepsilon)\right)
$$

for all $n \geq 1$ and $x, y \in X$. 
As a simplest example consider the Lebesgue measure and the ArnoldThom cat map of the torus $\mathbb{T}^{2}$, i.e., the linear automorphism of $\mathbb{T}^{2}$ given by the matrix $A=\left(\begin{array}{ll}2 & 1 \\ 1 & 1\end{array}\right)$. The same property is enjoyed by the Bowen-Margulis measures for Anosov systems.

This condition is rather strong. In particular, it implies that $\mu$ is a measure of maximal entropy and that

$$
h_{\mu}(f, x)=h_{\mu}(f)=h_{\mathrm{top}}(f)
$$

for all $x \in X$. Thus the multifractal spectrum of local entropies for a homogeneous measure is trivial:

$$
h_{\mathrm{top}}\left(f, K_{\alpha}\right)= \begin{cases}h_{\mathrm{top}}(f) & \text { if } \alpha=h_{\mathrm{top}}(f), \\ 0 & \text { otherwise. }\end{cases}
$$

Not surprisingly, the spectrum of correlation entropies is quite simple as well:

$$
H_{\mu}(f, q) \equiv h_{\text {top }}(f) \text {. }
$$

Obviously, $\mathcal{E}(\alpha)$ and $(1-q) H_{\mu}(f, q)$ are related by the Legendre transform. However, the interval $(\underline{\alpha}, \bar{\alpha})$ is empty. Thus, in this clearly degenerate situation, we can say that the multifractal formalism is valid for homogeneous measures. According to some definitions in the physics literature, homogeneous measures are not multifractal measures.

12.2. Gibbs measures for expansive homeomorphisms with specification. This is the most general situation known when the multifractal formalism for local entropies, according to Definition 10.5, is valid. Here we describe results obtained in [24].

First we introduce the notion of an expansive homeomorphism with specification and Gibbs measures.

Definition 12.2. A homeomorphism $f: X \rightarrow X$ is called expansive if there exists a constant $\gamma>0$ such that

$$
\text { if } \quad d\left(f^{n}(x), f^{n}(y)\right)<\gamma \text { for all } n \in \mathbb{Z} \text { then } \quad x=y \text {. }
$$

Such a $\gamma$ is called an expansivity constant.

Another important requirement is the following.

Definition 12.3 (see [5]). We say that a homeomorphism $f: X \rightarrow X$ has the specification property if for each $\delta>0$ there exists an integer $p=p(\delta)$ such that the following holds: if

(a) $I_{1}, \ldots, I_{n}$ are intervals of integers, $I_{j} \subseteq[a, b]$ for some $a, b \in \mathbb{Z}$ and all $j$,

(b) $\operatorname{dist}\left(I_{i}, I_{j}\right) \geq p(\delta)$ for $i \neq j$,

then for arbitrary $x_{1}, \ldots, x_{n} \in X$ there exists a point $x \in X$ such that 
(1) $f^{b-a+p(\delta)}(x)=x$,

(2) $d\left(f^{k}(x), f^{k}\left(x_{i}\right)\right)<\delta$ for $k \in I_{i}$.

We call such maps homeomorphisms with specification.

The specification property guarantees good mixing properties of $f$ and the existence of a sufficient number of periodic orbits.

We consider Gibbs measures for a special, but quite large, class $\mathcal{V}_{f}(X)$ of potentials, which is defined as follows. We say that $\varphi \in \mathcal{V}_{f}(X)$ if $\varphi$ is continuous and there exist $\varepsilon>0$ and $K>0$ such that for all $n \in \mathbb{N}$,

$$
\begin{aligned}
& d\left(f^{k}(x), f^{k}(y)\right)<\varepsilon \text { for } k=0, \ldots, n-1 \\
& \Rightarrow\left|\sum_{i=0}^{n-1} \varphi\left(f^{i}(x)\right)-\sum_{i=0}^{n-1} \varphi\left(f^{i}(y)\right)\right|<K .
\end{aligned}
$$

For example, for a hyperbolic diffeomorphism $f$ any Hölder continuous function $\varphi$ is in $\mathcal{V}_{f}(X)$ [14, Prop. 20.2.6].

The following theorem states the existence and basic properties of the equilibrium states for potentials in $\mathcal{V}_{f}(X)$. Basically it says that the equilibrium states exist and are unique, and moreover they are Gibbs measures.

THEOREM 12.4 (see $[5,11,14]$ ). If $f$ is an expansive homeomorphism with specification and $\varphi \in \mathcal{V}_{f}(X)$ then there exists a unique measure $\mu_{\varphi}$, called the equilibrium state of $\varphi$, such that

$$
P(\varphi)=h_{\mu_{\varphi}}(f)+\int \varphi d \mu_{\varphi},
$$

where $P(\varphi)$ is the topological pressure of $\varphi$. The equilibrium state $\mu_{\varphi}$ is a Gibbs measure, i.e., for sufficiently small $\varepsilon>0$ there exist constants $A_{\varepsilon}, B_{\varepsilon}>0$ such that for all $x \in X$ and $n \geq 0$,

$$
A_{\varepsilon} \leq \frac{\mu_{\varphi}\left(\mathcal{B}_{n}(x, \varepsilon)\right)}{\exp \left(-n P(\varphi)+\sum_{i=0}^{n-1} \varphi\left(f^{i}(x)\right)\right)} \leq B_{\varepsilon} .
$$

Moreover, $\mu_{\varphi}$ is ergodic, positive on open sets and mixing.

The characteristic property (12.1) of a Gibbs measure is the key which allows us to perform a complete multifractal analysis of local entropies. It is easy to see from (12.1) that Gibbs measures satisfy the entropy doubling condition. Therefore according to the results in the previous sections it makes sense to look at correlation entropies. The following result establishes the smoothness of correlation entropies as functions of the parameter $q$.

Lemma 12.5 (see [24]). Suppose $f: X \rightarrow X$ is an expansive homeomorphism with specification, $\varphi \in \mathcal{V}_{f}(X)$ and $\mu$ is the corresponding Gibbs measure. Then

$$
(1-q) H_{\mu}(f, q)=P(q \varphi)-q P(\varphi) .
$$


Moreover, $(1-q) H_{\mu}(f, q)$ is convex and continuously differentiable, and it is strictly convex provided $\mu$ is not a measure of maximal entropy.

The results from the previous section say that the Legendre transform of $T(q)=(1-q) H_{\mu}(f, q)$ give an upper estimate of the multifractal spectrum of local entropies. It turns out that there is an exact equality. The way to prove this, which is used in [24], was adopted from [1, 2] and consists in the following. It is quite clear that since the system $(f, \mu)$ is ergodic there is only one value $\alpha_{1}$ such that $\mu\left(K_{\alpha_{1}}\right)=1$, while for all other $\alpha$ 's the sets $K_{\alpha}$ have measure 0. Actually, we know from the Brin-Katok formula that $\alpha_{1}=h_{\mu}(f)$. Now, since $h_{\text {top }}\left(f, K_{\alpha}\right) \leq \alpha$ for all $\alpha \geq 0$ and $h_{\text {top }}\left(f, K_{\alpha}\right) \geq h_{\nu}(f)$ for every invariant measure $\nu$ such that $\nu\left(K_{\alpha}\right)=1$ (Theorem 3.2), taking $\alpha_{1}=h_{\mu}(f)$ and $\nu=\mu$ we immediately conclude that

$$
\alpha_{1} \geq h_{\mathrm{top}}\left(F, K_{\alpha_{1}}\right) \geq h_{\mu}(f)=\alpha_{1} .
$$

Hence, $h_{\text {top }}\left(F, K_{\alpha_{1}}\right)=\alpha_{1}$.

It turns out that we can generalize this argument to all other $\alpha$ 's. The idea is to introduce a one-parameter family of measures $\mu_{q}$, which are Gibbs states for the potential $q \varphi$. Then for a suitable parameterization $\alpha(q)$,

$$
\mu_{q}\left(K_{\alpha}\right)= \begin{cases}1 & \text { if } \alpha=\alpha(q) \\ 0 & \text { otherwise. }\end{cases}
$$

This parameterization $\alpha(q)$ is build in the following way. Consider $T(q)=$ $P(q \varphi)-q P(\varphi)=(1-q) H_{\mu}(f, q)$. It turns out to be a differentiable decreasing function of $q$. Let $\alpha(q)=-T^{\prime}(q) \geq 0$ and $\mu_{q}$ be an equilibrium state for $\varphi_{q}=q \varphi$. Comparing (12.1) for two Gibbs measures $\mu$ and $\mu_{q}$ corresponding to the potentials $\varphi$ and $q \varphi$ we see that for every $x \in X$,

$$
h_{\mu_{q}}(f, x)=T(q)+q h_{\mu}(f, x)
$$

provided at least one of the local entropies $h_{\mu}(f, x), h_{\mu_{q}}(f, x)$ exists. Therefore, if $x \in K_{\alpha(q)}$, i.e., $h_{\mu}(f, x)=\alpha(q)$, then

$$
h_{\mu_{q}}(f, x)=T(q)+q \alpha(q) .
$$

It is not very difficult to verify that the measure-theoretic entropy of $\mu_{q}$ is given by

$$
h_{\mu_{q}}(f)=T(q)+q \alpha(q) .
$$

Thus, since $h_{\mu_{q}}(f, x)=h_{\mu_{q}}(f)$ for $\mu_{q}$-a.e. $x$, and the equality holds only for $x \in K_{\alpha(q)}$, one gets $\mu_{q}\left(K_{\alpha(q)}\right)=1$. The final argument is similar to the one above for $\alpha=h_{\mu}(f)$. Namely, from Theorem 10.4 we know that for all $\alpha \geq 0$,

$$
h_{\mathrm{top}}\left(f, K_{\alpha}\right) \leq T^{*}(\alpha)=\inf _{q}(T(q)+q \alpha) .
$$

On the other hand, since $\mu_{q}$ is an invariant measure concentrated on $K_{\alpha(q)}$, 
we obtain

$$
h_{\text {top }}\left(f, K_{\alpha(q)}\right) \geq h_{\mu_{q}}(f)=T(q)+q \alpha(q) .
$$

Comparing the last two formulas we conclude that $h_{\text {top }}\left(f, K_{\alpha(q)}\right)=T^{*}(\alpha(q))$ for all $q$. To finish the proof we have to apply the result from [22] which states that $\{\alpha(q)\}$ for $q \in \mathbb{R}$ exhausts the domain of the multifractal spectrum. More precisely, there exists an interval $[\underline{\alpha}, \bar{\alpha}]$ such that

$$
K_{\alpha}=\emptyset \quad \text { for } \alpha \notin[\underline{\alpha}, \bar{\alpha}],
$$

for every $\alpha \in(\underline{\alpha}, \bar{\alpha})$ there exists $q \in \mathbb{R}$ such that $\alpha=\alpha(q)$.

This means that we obtained a complete description of the multifractal spectrum of local entropies for an expansive homeomorphism with specification.

12.3. Weak Gibbs measures for interval maps with indifferent fixed points. For simplicity we consider a piecewise monotonic map with two full branches, expanding everywhere except one fixed point. The precise conditions are given in the next definition. This, and even more general situations, are considered in [13, 20, 28].

Definition 12.6. We say that $f:[0,1] \rightarrow[0,1]$ is a Manneville-Pomeau type map if

(1) $f$ is piecewise monotonic and has two full branches, i.e., there exists $p>0$ such that $f(0, p)=(0,1)$ and $f(p, 1)=(0,1)$;

(2) the branches $\left.f\right|_{(0, p)}$ and $\left.f\right|_{(p, 1]}$ are $C^{2}$;

(3) $f^{\prime}(x)>1$ for all $x>0$ and $f^{\prime}(x) \geq \lambda>1$ for $x \in(p, 1)$.

(4) $f$ has the following asymptotic behavior as $x \rightarrow 0_{+}$:

$$
f(x)=x+C x^{1+\gamma}(1+u(x))
$$

with constants $C>0, \gamma \in(0,1)$, and $u(x)$ a $C^{1}$ function such that $u^{\prime}(x)=$ $\mathcal{O}\left(x^{t-1}\right)$ as $x \rightarrow 0_{+}$, for some $t>0$.

G. Pianigiani [19] was the first to show the existence of an absolutely continuous invariant measure for Manneville-Pomeau type maps. We denote this measure by $\mu$. This invariant measure has positive entropy, which is given by Rokhlin's formula

$$
h_{\mu}(f)=\int \log \left|f^{\prime}\right| d \mu .
$$

The ergodic properties of $\mu$ have been studied extensively in the literature (see, e.g., $[13,15,27,12])$. In particular, it has recently been established that for these systems correlations decay polynomially. Note that for expanding systems the decay is exponential.

The absolutely continuous invariant measure $\mu$ is an equilibrium state for the potential $\varphi=-\log \left|f^{\prime}\right|$. But it is not unique: the Dirac measure concentrated at 0 is an equilibrium state as well. The existence of two equilibrium states (i.e., non-uniqueness of equilibrium states) results in the non-smooth 
behavior of the pressure function $P(q \varphi)$. Combining the results from $[20,25]$ we obtain the following statement.

Lemma 12.7. Let $f$ be a Manneville-Pomeau type map. Then $P(q \varphi)$ is a continuous, convex, non-increasing function. Moreover, $P(q \varphi)=0$ for $q \geq 1$, and $P(q \varphi)>0$ for $q<1, P(q \varphi)$ is a real-analytic function of $q<1$. At the critical point one has the asymptotics

$$
\frac{P(q \varphi)}{1-q} \rightarrow h_{\mu}(f) \quad \text { as } q \nearrow 1 .
$$

The equilibrium state $\mu$ is not a Gibbs measure according to the standard definitions in ergodic theory (e.g., Theorem 12.4). The quotient

$$
\frac{\mu\left(\mathcal{B}_{n}(x, \varepsilon)\right)}{\exp \left(\sum_{k=0}^{n-1} \varphi\left(f^{k}(x)\right)\right)}
$$

is not uniformly bounded from above and below by constants independent of $n$. However, for every sufficiently small $\varepsilon>0$ one can find a subexponential sequence $K_{n}$, i.e., $\lim _{n \rightarrow \infty}\left(\log K_{n}\right) / n=0$, such that

$$
\frac{1}{K_{n}} \leq \frac{\mu\left(\mathcal{B}_{n}(x, \varepsilon)\right)}{\exp \left(\sum_{k=0}^{n-1} \varphi\left(f^{k}(x)\right)\right)} \leq K_{n}
$$

for all $x$. This observation leads to the following definition.

Definition 12.8. We say that $\mu$ is a weak Gibbs measure if for every sufficiently small $\varepsilon>0$ one can choose $K_{n}$ with $\lim _{n \rightarrow \infty}\left(\log K_{n}\right) / n=0$ such that (12.2) holds for every $x$.

The estimate (12.2) does not actually imply that $\mu$ satisfies the entropy doubling condition. Though it is clear that $\mu$ satisfies a weaker version of the doubling condition:

Definition 12.9. We say that $\mu$ satisfies the weak entropy doubling condition if for every sufficiently small $\varepsilon>0$ one has

$$
\lim _{n \rightarrow \infty} \frac{1}{n} \log \sup _{x} \frac{\mu\left(\mathcal{B}_{n}(x, \varepsilon)\right)}{\mu\left(\mathcal{B}_{n}(x, \varepsilon / 2)\right)}=0 .
$$

It is easy to check that after appropriate modifications in the proofs all the results in Section 10 remain valid. For example, from (12.3) we conclude that for every sufficiently small $\varepsilon>0$ and any $\delta>0$ there exist $N$ such that for all $n>N$ one has

$$
C(\varepsilon, n)=\sup _{x} \frac{\mu\left(\mathcal{B}_{n}(x, \varepsilon)\right)}{\mu\left(\mathcal{B}_{n}(x, \varepsilon / 2)\right)} \leq \exp (\delta n) .
$$

Therefore, the constants $C_{1}, C_{2}$ from the statement of Lemma 10.1 should be replaced by some numbers $C_{1}(n), C_{2}(n)$, which do not decay or grow too fast with $n$. In particular, there exists a constant $K>0$, independent of $n$ 
and $\delta$, such that $C_{1}(n)>\exp (-K \delta n)$ and $C_{2}(n)<\exp (K \delta n)$ for sufficiently large $n$. As a result, for $q>1$ we obtain

$$
\begin{aligned}
\overline{C h}_{\mu}(f, q, X, 2 \varepsilon)-K \delta & \leq \varlimsup_{n \rightarrow \infty} \frac{1}{n} \log \int \mu\left(\mathcal{B}_{n}(x, \varepsilon)\right)^{q-1} d \mu \\
& \leq \overline{C h}_{\mu}(f, q, X, \varepsilon)+K \delta .
\end{aligned}
$$

Since $\delta$ is an arbitrary positive number, we obtain exactly the same result as in Lemma 10.3.

As a result we conclude that the Legendre transform of $(1-q) H_{\mu}(f, q)=$ $P(q \varphi)$ gives an upper estimate on the multifractal spectrum of local entropies.

The problem is now of course to show that we actually have equality of the multifractal spectrum and the Legendre transform of $(1-q) H_{\mu}(f, q)$. The method used in the previous example employs the construction of "reference measures" $\mu_{q}$. We have used the equilibrium states corresponding to the potentials $q \varphi$ as such measures. In this case we can repeat the same argument for $q \leq 1$ (corresponding to $\alpha \geq h_{\mu}(f)$ ) and achieve the same result. However, for $q>1$ (corresponding to $\alpha<h_{\mu}(f)$ ), the equilibrium states for $\varphi_{q}=q \varphi$ are just the Dirac measures, carrying zero entropy and therefore useless for our purposes.

At the present moment it is not clear what the structure of the multifractal spectrum for $\alpha<h_{\mu}(f)$ is. To answer this question, in our opinion, one should study in greater detail the pre-measures involved in the definition of topological entropy.

13. Concluding remarks. In the first part of this paper we have constructed a general formalism which allows us to obtain an upper estimate on the multifractal spectrum of local entropies. Under additional assumptions (the entropy doubling condition) we have shown that the Legendre transform of the spectrum of correlation entropies gives an upper estimate. We have illustrated these results on three examples. For homogeneous measures the multifractal spectrum is a delta-like function. In the case of Gibbs measures for expansive homeomorphisms with specification the multifractal spectrum is a smooth strictly concave function, which is equal to the Legendre transform of $(1-q) H_{\mu}(f, q)$. For non-uniformly hyperbolic dynamical systems (interval maps with indifferent fixed points) the methods developed recover the multifractal spectrum only partially.

Acknowledgments. The second author is grateful to Lars Olsen for bringing the results from [16] to his attention. He also acknowledges the support of the Dutch Organization for Scientific Research (NWO), grant 613-06551. 


\section{References}

[1] L. Barreira, Ya. Pesin, and J. Schmeling, Multifractal spectra and multifractal rigidity for horseshoes, J. Dynam. Control Systems 3 (1997), 33-49.

[2] - - - - - On a general concept of multifractality: multifractal spectra for dimensions, entropies, and Lyapunov exponents. Multifractal rigidity, Chaos 7 (1997), 27-38.

[3] C. Beck and F. Schlögl, Thermodynamics of Chaotic Systems, Cambridge Univ. Press, Cambridge, 1993.

[4] R. Bowen, Topological entropy for noncompact sets, Trans. Amer. Math. Soc. 184 (1973), 125-136.

[5] —, Some systems with unique equilibrium states, Math. Systems Theory 8 (1974//75), 193-202.

[6] M. Brin and A. Katok, On local entropy, in: Geometric Dynamics (Rio de Janeiro, 1981), Lecture Notes in Math. 1007, Springer, Berlin, 1983, 30-38.

[7] K. Falconer, Fractal Geometry, Wiley, Chichester, 1990.

[8] P. Grassberger and I. Procaccia, Dimensions and entropies of strange attractors from a fluctuating dynamics approach, Phys. D 13 (1984), 34-54.

[9] M. Guysinsky and S. Yaskolko, Coincidence of various dimensions associated with metrics and measures on metric spaces, Discrete Contin. Dynam. Systems 3 (1997), 591-603.

[10] T. C.Halsey, M. H. Jensen, L. P. Kadanoff, I. Procaccia, and B. I. Shraiman, Fractal measures and their singularities: the characterization of strange sets, Phys. Rev. A (3), 33 (1986), 1141-1151.

[11] N. T. A. Haydn and D. Ruelle, Equivalence of Gibbs and equilibrium states for homeomorphisms satisfying expansiveness and specification, Comm. Math. Phys. 148 (1992), 155-167.

[12] H. Hu, Decay of correlations for piecewise smooth maps with indifferent fixed points, preprint, 1998.

[13] S. Isola, Dynamical zeta functions and correlation functions for non-uniformly hyperbolic transformations, preprint, 1995.

[14] A. Katok and B. Hasselblatt, Introduction to the Modern Theory of Dynamical Systems, Encyclopedia Math. Appl. 54, Cambridge Univ. Press, Cambridge, 1995.

[15] C. Liverani, B. Saussol, and S. Vaienti, A probabilistic approach to intermittency, preprint.

[16] L. Olsen, A multifractal formalism, Adv. Math. 116 (1995), 82-196.

[17] Ya. B. Pesin, Dimension Theory in Dynamical Systems, Univ. of Chicago Press, Chicago, IL, 1997.

[18] Ya. B. Pesin and B. S. Pitskel', Topological pressure and the variational principle for noncompact sets, Funktsional. Anal. i Prilozhen. 18 (1984), no. 4, 50-63 (in Russian).

[19] G. Pianigiani, First return map and invariant measures, Israel J. Math. 35 (1980), 32-48.

[20] T. Prellberg and J. Slawny, Maps of intervals with indifferent fixed points: thermodynamic formalism and phase transitions, J. Statist. Phys. 66 (1992), 503-514. 
[21] A. W. Roberts and D. E. Varberg, Convex Functions, Academic Press, New York, 1973.

[22] J. Schmeling, On the completeness of multifractal spectra, preprint WIAS, Berlin, 1996.

[23] F. Takens and E. Verbitski, Generalized entropies: Rényi and correlation integral approach, Nonlinearity 11 (1998), 771-782.

[24] -, 一, Multifractal analysis of local entropies for expansive homeomorphisms with specification, Comm. Math. Phys. 203 (1999), 593-612.

[25] M. Urba/nski, Parabolic Cantor sets, Fund. Math. 151 (1996), 241-277.

[26] T. Ward, Entropy of Compact Group Automorphisms, lecture notes, 1994.

[27] L.-S. Young, Recurrence times and rates of mixing, preprint, 1997.

[28] M. Yuri, Thermodynamic formalism for certain nonhyperbolic maps, preprint.

Department of Mathematics

University of Groningen

P.O. Box 800

9700 AV, Groningen, The Netherlands

E-mail: f.takens@math.rug.nl

e.verbitski@math.rug.nl

Received 19 October 1999;

in revised form 21 March 2000 